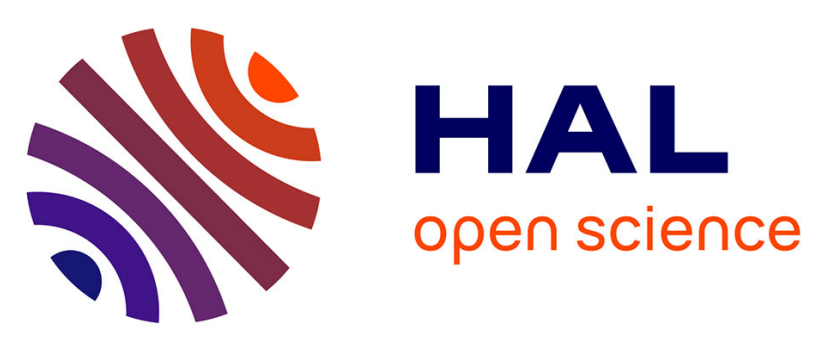

\title{
A particle-tracking formulation of advective-diffusive heat transport in deformable fracture networks
}

Silvia De Simone, Benoît Pinier, Olivier Bour, Philippe Davy

\section{To cite this version:}

Silvia De Simone, Benoît Pinier, Olivier Bour, Philippe Davy. A particle-tracking formulation of advective-diffusive heat transport in deformable fracture networks. Journal of Hydrology, 2021, 603 (D), pp.127157. 10.1016/j.jhydrol.2021.127157 . insu-03431972

\section{HAL Id: insu-03431972 \\ https://hal-insu.archives-ouvertes.fr/insu-03431972}

Submitted on 17 Nov 2021

HAL is a multi-disciplinary open access archive for the deposit and dissemination of scientific research documents, whether they are published or not. The documents may come from teaching and research institutions in France or abroad, or from public or private research centers.
L'archive ouverte pluridisciplinaire HAL, est destinée au dépôt et à la diffusion de documents scientifiques de niveau recherche, publiés ou non, émanant des établissements d'enseignement et de recherche français ou étrangers, des laboratoires publics ou privés. 


\section{Journal Pre-proofs}

Research papers

A particle-tracking formulation of advective-diffusive heat transport in deformable fracture networks

Silvia De Simone, Benoît Pinier, Olivier Bour, Philippe Davy

PII:

S0022-1694(21)01207-5

DOI: https://doi.org/10.1016/j.jhydrol.2021.127157

Reference:

HYDROL 127157

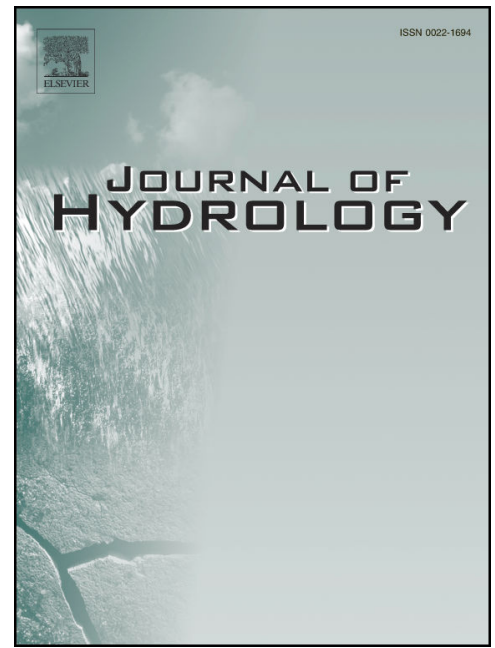

To appear in:

Journal of Hydrology

Received Date:

10 September 2021

Accepted Date:

1 November 2021

Please cite this article as: Simone, S.D., Pinier, B., Bour, O., Davy, P., A particle-tracking formulation of advective-diffusive heat transport in deformable fracture networks, Journal of Hydrology (2021), doi: https:// doi.org/10.1016/j.jhydrol.2021.127157

This is a PDF file of an article that has undergone enhancements after acceptance, such as the addition of a cover page and metadata, and formatting for readability, but it is not yet the definitive version of record. This version will undergo additional copyediting, typesetting and review before it is published in its final form, but we are providing this version to give early visibility of the article. Please note that, during the production process, errors may be discovered which could affect the content, and all legal disclaimers that apply to the journal pertain.

(C) 2021 Published by Elsevier B.V. 


\section{Highlights}

A particle-tracking formulation of advective-diffusive heat transport in deformable fracture networks

Silvia De Simone, Benoît Pinier, Olivier Bour, Philippe Davy

- We solve heat transport with thermo-mechanical (TM) deformation in fractured systems

- Particle tracking is combined with semi-analytical solutions

- Fluid/rock thermal diffusion is modeled through a memory diffusive heat exchange

- TM rock deformation and fracture aperture variation are analytically estimated

- The consequent variation of flow velocity significantly impacts the heat transfer 


\title{
A particle-tracking formulation of advective-diffusive heat transport in deformable fracture networks
}

\author{
Silvia De Simone ${ }^{\mathrm{a}, *}$, Benoît Pinier ${ }^{\mathrm{b}}$, Olivier Bour ${ }^{\mathrm{a}}$, Philippe Davy ${ }^{\mathrm{a}}$ \\ ${ }^{a}$ Univ Rennes, CNRS, Géosciences Rennes, UMR 6118, 35000 Rennes, France \\ ${ }^{b}$ Itasca Consultants SAS, Rennes, France
}

\begin{abstract}
Modeling heat transfer in complex heterogeneous fractured system is key for geothermal energy applications. Discrete fracture network (DFN) modeling is the classical framework to reproduce the advective part of the transport, which is determined by the fracture connectivity and heterogeneity. This approach in general sacrifices the representation of the rock matrix, disregarding both its diffusive heat exchange with the fractures and the effects of its thermo-mechanical deformation on the fracture aperture. Here we propose a new semi-analytic formulation that can be implemented in a DFN simulator with particle tracking approach. The contribution of the rock matrix in terms of diffusive heat exchange and thermal contraction/expansion is analytically evaluated, which respectively impact the advective heat transfer and the fracture aperture variation. The method is proved to be accurate and robust. Results from simulations of cold fluid injection show that rock contraction affects the transmissivity, which accelerates the advective transport resulting in a faster recovery of cold fluid at the outlet. The methodology enables investigating the reservoir behavior and optimizing the geothermal performance while keeping the computational effort within reasonable values. This allows exploring the uncertainty in cases when the in-situ characterization is poor, which is the spirit of the DFN modeling.
\end{abstract}

Keywords: heat transport, particle tracking, discrete fracture network, geothermal systems, thermo-hydro-mechanical coupling

\section{Introduction}

Deep geothermal energy represents a powerful and clean energy prospect, with the potential to generate huge and virtually unlimited energy (Giardini, 2009; Tester et al., 2007). Geothermal plants generally involve the circulation of fluids into hot reservoirs located in the crystalline basement, characterized by a very low permeability of the rock mass and a complex system of preexisting natural fractures (Jung, 2013). Enhancement of fracture transmissivity is often performed by means of massive fluid injection during the preliminary stimulation stage, leading to the development of the so-called Enhanced Geothermal Systems (EGS). Both before and after the stimulation operations, a full characterization of the rock and fracture properties is difficult, which increases the uncertainty on the heat extraction performance. In this context, numerical modeling is a key instrument to forecast the potential heat production of geothermal reservoirs under a number of scenarios (Baria et al., 1999).

Heat transfer in fractured reservoirs is driven by fluid advection through the fracture network and diffusion in the host rock. The first process defines the spatial extension of the affected domain, while the second one controls the fluid-solid heat exchange and, therefore, the geothermal performance (Bruel, 2002). The behavior may be complex because the two processes are coupled and because of the heterogeneity of the flow velocity and fracture aperture fields in the network (Becker and

\footnotetext{
${ }^{*}$ Corresponding author

Email address: silviadesi@gmail.com (Silvia De Simone )
}

Shapiro, 2003). The complexity is further increased when thermal deformations are taken into account. Long-term fluid circulation leads to the cooling and consequent contraction of the host rock which tends to increase the fracture aperture (Koh et al., 2011). The variation of the fracture aperture has two effects. On the one hand, it affects the fluid-filled fracture volume that exchanges heat with the rock through the fracture surface, and therefore the exchanged heat. On the other hand, it may imply a variation of the flow velocity which in turn affects the heat advective transport, slowing down or speeding up the heat production. A potential consequence is the opening of new fast flow paths that may short-circuit the connection between the paired injection and extraction wells, with negative impacts on the geothermal production (McDermott et al., 2006). Quantifying these processes is crucial for the design and optimization of heat extraction. However, modeling these processes in heterogeneous fractured systems poses a number of numerical challenges, because they occur on very different length and time scales, and because of the large variability of fracture size and transmissivity (Bonnet et al., 2001; Bour and Davy, 1997, 1999; de Dreuzy et al., 2001; Davy et al., 2010).

Continuum-based approaches accurately reproduce the fully coupled thermo-hydro-mechanical (THM) process, but they are not able to reproduce the intricate structure of fractured reservoir, unless the fracture system is dense enough to be represented as an equivalent porous medium. Discrete fracture network (DFN) modeling is the most appropriate framework to reproduce the advective part of the problem, which is determined by the fracture connectivity and heterogeneity. However, the 
representation of the diffusive heat exchange with the rock matrix and of the thermo-mechanical (TM) coupling suffers from some limitations (Jing, 2003). A number of DFN-based approaches focuses on the advective transport in the network of connected fractures, disregarding the effects of the rock matrix (e.g., Frampton and Cvetkovic, 2011; Hyman et al., 2019). However, the diffusive exchange between fracture and rock is recognized as playing a key role in all transport problems (Carrera et al., 1998), and in particular in the case of heat transfer. Moreover, the mechanical response of fractures to heat is due to the rock thermal expansion or contraction. Thus, it depends on the portion of the rock matrix affected by thermal diffusion and on the rock properties. Discrete fracture-matrix (DFM) models reproduce the behavior of fractures embedded in a rock matrix through explicit representation of rock blocks (e.g., Hu et al., 2017; Odsæter et al., 2019; Flemisch et al., 2018). Although this approach is accurate, and it may be combined with the numerical simulation of the thermal deformations, it incurs high computational cost which limits the number of fractures that may be explicitly represented or the number of scenarios that may be analyzed (see Lei et al., 2017, for a comprehensive review).

To represent the exchange with the rock, the assumption of a diffusive heat flux perpendicular to the fracture plane is widely adopted, which can be solved analytically. The adoption of analytic solutions greatly reduces the computational effort, which allows for stochastic modeling of large three-dimensional reservoirs with thousands of fractures. Several authors have combined Lagrangian particle-tracking solvers and analytic solutions of the diffusion process to reproduce transport of solute mass of chemical species (Dagan et al., 1992; Cvetkovic et al., 1999; Painter and Cvetkovic, 2005). The framework has been recently applied to heat transport by Gisladottir et al. (2016). In many cases the approach is limited by some underlying assumptions. The first is the one of infinite matrix, which is reasonable only for short time or large fracture spacing. The second is that the trajectories are stationary in time and independent on each other. In addition, the TM coupling is rarely contemplated in this context.

In this paper, we present a new simplified methodology to simulate heat transfer in fractured media taking into account the diffusive exchange with the rock matrix and the consequent thermo-mechanical fracture aperture variation due to rock deformation. The methodology is based on a particle tracking approach and it is similar to the method of the characteristics (Konikow and Bredehoeft, 1978). The contribution of the rock matrix in terms of diffusive heat exchange and thermal contraction/expansion is analytically evaluated, which respectively impact the heat transfer and the fracture aperture variation. This latter in turns affects the heat transfer and the velocity field, which is updated in time assuming quasi-steady state conditions. We disregard the effects of the pore-pressure variations on the fracture aperture and in the rock (hydro-mechanical (HM) coupling). However, we do take into account the impact of the flow velocity on the heat transfer, as well as the effects of the temperature on the fracture aperture, and the impact of the aperture variation on the flow velocity. We therefore describe this approach as a model for simulating $\mathrm{T}(\mathrm{H}) \mathrm{M}$ coupling. Given its semi-analytic nature, this formulation allows reproducing heat transport in temperature-dependent complex fracture networks while keeping the computational time within reasonable values.

In the following, we first introduce the conceptual model and summarize the governing equations. Then, we show the classical analytic solution for a single trajectory with constant in time fracture aperture and velocity. Afterwards, we present the detail of the proposed semi-analytic method for single trajectory with temperature-dependent fracture aperture and velocity. We show that extending the second approach to the case of multiple trajectories allows us to deal with non-stationary trajectories in a changing environment. After presenting the details of the method, its implementation and validation, we compare the behavior for constant and non-constant fracture aperture and velocity, for the case of a single trajectory, and we discuss the numerical issues and the implications. Finally, we show results for a DFN and we discuss the effects of non-stationary trajectories on the observed temperatures.

\section{Governing equations and assumptions}

Let a heterogeneous fracture network be composed by connected fractures embedded in a rock matrix. We assume that each fracture is planar and perfectly symmetric with respect to its central axis and thus we refer to the semi-volume (Fig. 1). The fracture half-aperture $b(\mathbf{x}, t)$ is variable in time and space, where $\mathbf{x}$ represents the coordinate vector in the fracture plane and $t$ is time. The variability in space is smooth such that we can keep the assumption of planar fracture as valid. No distinction is made between hydraulic aperture and mechanical aperture, and fracture roughness is disregarded. We focus on the thermomechanic processes occurring for long-term non-isothermal injection and we do not consider the fracture aperture variations due to pressure increase, which mostly occur during the stimulation phase or at the beginning of injection operations (Baria et al., 1999). Fracture failure with either opening (mode I) or shearing (mode II) mechanism is not contemplated here, and linear elastic behavior is assumed. Creation of new fractures or propagation of the pre-existing ones are also neglected.

This conceptual model is used in the following to illustrate the governing equations for flow, heat transport and mechanical deformation with respect to a single fracture, and it is successively extended to the case of a streamline through a fracture network.

\subsection{Fluid flow}

We consider that fractures are fluid saturated, and that fluid flow occurs exclusively in the fracture network, while the rock matrix is impermeable. Conservation of fluid mass for unit volume of fracture gives the flow equation for the fracture

$$
\frac{\partial b(\mathbf{x}, t)}{\partial t}+\nabla[b(\mathbf{x}, t) \mathbf{v}(\mathbf{x}, t)]+W=0,
$$

where we have assumed homogeneous fluid density and incompressible fluid. The term $W$ represents a sink/source, while 


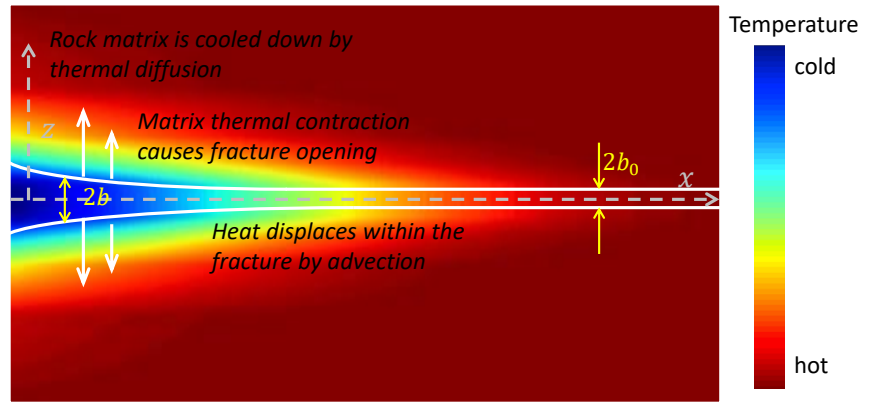

Figure 1: Conceptual model for heat transport and thermo-mechanical response in one fracture embedded in a rock matrix, subject to injection of a cold fluid. The opposite behavior is observed in the case of injection of a hot fluid, i.e., the rock matrix expands, and the fracture aperture reduces. Note that the rock matrix is assumed as impervious, so flow and advective transport only occurs in the fracture, while the fluid in the fracture exchanges heat with the rock matrix by thermal diffusion. Figure not to scale.

$\mathbf{v}(\mathbf{x}, t)$ is the fluid flow velocity vector that is proportional to the pressure gradient $\nabla p$. Assuming the validity of the cubic law, with the fracture hydraulic aperture coinciding with the mechanical aperture and equal to $2 b$, it reads

$$
\mathbf{v}(\mathbf{x}, t)=-\frac{4 b(\mathbf{x}, t)^{2}}{12 \mu} \nabla p(\mathbf{x}, t),
$$

where $\mu$ is the fluid viscosity. The boundary conditions (BCs) at the inlet can be either of imposed pressure or flow rate. In the following we assume that the fracture aperture variation is slow, which allows for considering quasi-steady state conditions for flow, i.e., the velocity field does not change over a certain time. Under this condition, the first term of eq. (1) vanishes.

\subsection{Heat transport}

We consider that heat transfer occurs by fluid advection within the fracture system and by thermal diffusion within the rock matrix, mostly in the direction orthogonal to the fracture. Lateral diffusion is disregarded both in the fracture and in the matrix, but we will partly introduce this effect in the following. We also assume infinite diffusive matrix and flat temperature profile within the fracture thickness. According to this setting, the heat transport within the fracture is described by the energy balance for an arbitrary semi-volume of fracture (Carrera et al., 1998)

$$
b(\mathbf{x}, t) C \frac{\partial T(\mathbf{x}, t)}{\partial t}+b(\mathbf{x}, t) C \mathbf{v}(\mathbf{x}, t) \nabla T(\mathbf{x}, t)=-J(\mathbf{x}, t)
$$

where $C$ is the fluid heat capacity, assumed as constant and $T(\mathbf{x}, t)$ represents the fluid temperature in the fracture. $J(\mathbf{x}, t)$ is the diffusive heat flux that the fluid exchanges with the rock matrix per unit surface, which is defined as

$$
J(\mathbf{x}, t)=-\left.\kappa \frac{\partial T_{m}(\mathbf{x}, z, t)}{\partial z}\right|_{z=b},
$$

where $\kappa$ is the rock thermal conductivity, assumed as constant, and $z$ is the coordinate in the direction normal to the fracture, with origin at the fracture center. The temperature in the matrix $T_{m}(\mathbf{x}, z, t)$ is given by the energy balance

$$
C_{m} \frac{\partial T_{m}(\mathbf{x}, z, t)}{\partial t}=\kappa \frac{\partial^{2} T_{m}(\mathbf{x}, z, t)}{\partial z^{2}}
$$

for $b \leq z<+\infty$. $C_{m}$ represents the rock heat capacity, assumed as constant.

Equations (3) and (5) are coupled by means of eq. (4) and they have to be solved together. Another coupling condition is provided by the temperature continuity at the fracture wall, i.e., the fluid temperature in the fracture is equal to the rock temperature at the fracture edge, which defines a BC for eq. (5),

$$
T_{m}(\mathbf{x}, b, t)=T(\mathbf{x}, t), \forall(\mathbf{x}, t) .
$$

The other BC for eq. (5) is given by the hypothesis of infinite matrix. Without any loss of generality, we assume that the initial temperature is uniform in the system and equal to 0 , both in the fractures and in the rock. Different initial conditions are easily reproduced by considering the temperature values as temperature variations. We want to solve the problem of continuous imposed fluid temperature at one extreme of the fracture, $\mathbf{x}=\mathbf{x}_{0}$, which is also considered as infinite in the longitudinal direction. Therefore, the initial and boundary conditions for the matrix and the fracture are the following

$$
\begin{array}{rll}
T_{m}(\mathbf{x}, z, 0)=0 & , & T(\mathbf{x}, 0)=0 \\
T_{m}(\mathbf{x}, b, t)=T(\mathbf{x}, t) & , & T\left(\mathbf{x}_{0}, t\right)=T_{0}(t) \\
T_{m}(\mathbf{x}, \infty, t)=0 & , & T(\infty, t)=0 .
\end{array}
$$

\subsection{Mechanical deformation}

The rock matrix behaves as a linear elastic material, which under non-isothermal conditions is governed by the constitutive law (Timoshenko and Goodier, 1951)

$$
\boldsymbol{\sigma}=2 G \boldsymbol{\varepsilon}+\lambda \varepsilon_{v o l} \mathbf{I}-(2 G+3 \lambda) \alpha_{T} T_{m} \mathbf{I}
$$

where $\sigma$ is the stress tensor, $G$ and $\lambda$ are the shear and Lamé moduli, respectively, $\alpha_{T}$ is the linear thermal expansion coefficient, $\mathbf{I}$ is the identity matrix, $\boldsymbol{\varepsilon}=1 / 2\left[\nabla \mathbf{u}+(\nabla \mathbf{u})^{T}\right]$ is the strain tensor and $\varepsilon_{v o l}=\nabla \cdot \mathbf{u}$ is the volumetric strain, which corresponds to $\varepsilon_{x}+\varepsilon_{y}+\varepsilon_{z}=\partial u_{x} / \partial x+\partial u_{y} / \partial y+\partial u_{z} / \partial z$, where $\mathbf{u}$ is the displacement vector. Stresses, strains and displacements evolve in time in response to changes in temperature. The stress tensor refers to stress variation if an initial stress $\neq 0$ is assumed. We assume the sign convention such that tensile stress and extensional strain are positive. The $x$ and $y$ axes are taken as respectively parallel and transverse to the flow direction in the fracture plane, while the $z$ axis is perpendicular to the fracture wall.

The mechanical equilibrium, in absence of body and surface forces, is therefore expressed by

$$
G \nabla^{2} \mathbf{u}+(G+\lambda) \nabla(\nabla \cdot \mathbf{u})=(2 G+3 \lambda) \alpha_{T} \nabla\left(T_{m}\right),
$$

where we observe that the deformation is driven by the temperature gradient. Note that the effects of the pore pressure variation is neglected as the rock matrix is assumed as impermeable. 
Considering a uniform temperature profile (zero gradient) in the fracture in-plane transverse direction, we can assume plane strain conditions in the y-direction, i.e., $\varepsilon_{y}=0$. The deformations in the direction longitudinal to the fracture are disregarded because they are small given the temperature distribution. We also assume free thermal deformation in the direction transverse to the fracture into the matrix, which implies that the corresponding compressive stress is zero, i.e., $\sigma_{z}=0$. Furthermore, we disregard the shear stress $\tau_{x z}$ based on the assumption that the temperature gradient is smooth in the longitudinal direction. We test and discuss these assumptions in Section 5.3. Under these hypothesis, the problem is greatly simplified into the one-dimensional equilibrium equation along the direction $z$, for each location $\mathbf{x}$, such as eq. (9) translates into

$$
\frac{\partial^{2} u_{z}(\mathbf{x}, z, t)}{\partial z^{2}}=\frac{1+v}{1-v} \alpha_{T} \frac{\partial T_{m}(\mathbf{x}, z, t)}{\partial z}, b \leq z<+\infty
$$

where $v$ is the Poisson ratio and the following relation holds, $(1+v) /(1-v)=(2 G+3 \lambda) /(2 G+\lambda)$. The displacement $u_{z}$ is found by solving eq. (10) under the boundary conditions of free displacement (i.e., zero stress) at $z=b$ and infinite rock matrix, i.e., zero displacement at $z=\infty$.

At each fracture location and time, the variation of the semi-aperture with respect to the initial conditions, $\Delta b(\mathbf{x}, t)=$ $b(\mathbf{x}, t)-b(\mathbf{x}, 0)$, is equal to the displacement undergone by the fracture wall in the direction perpendicular to the fracture, $u_{z}(\mathbf{x}, b, t)$, such as

$$
\Delta b(\mathbf{x}, t)=u_{z}(\mathbf{x}, b, t)=-\int_{b}^{\infty} \frac{1+v}{1-v} \alpha_{T} T_{m}(\mathbf{x}, z, t) \mathrm{d} z .
$$

Note that the expression corresponds to the integral over space of the rock matrix thermal deformation $\varepsilon_{z}=\partial u_{z} / \partial z$, because we assume that the displacement is 0 at $z=\infty$, where the temperature variation is zero.

\section{Solutions for a single flow path}

\subsection{Single flow path with constant fracture aperture and flow velocity}

Assume a single flow path with longitudinal coordinate $\ell$ parallel to the flow direction and constant in time fracture aperture, such as $b=b(\ell)$. In this framework, the fluid flow is steady state and the velocity is constant and equal to $v(\ell)$. The energy balance of eq. (3) is therefore simplified into

$$
\frac{\partial T(\ell, t)}{\partial t}+v(\ell) \frac{\partial T(\ell, t)}{\partial \ell}=-\frac{J(\ell, t)}{C b(\ell)}
$$

This problem can be solved analytically, as proposed by several authors (e.g., Liu et al., 2007) and as we summarize in Appendix Appendix A. The temperature evolution in the fluid and in the rock matrix in the case of constant temperature $\mathrm{BC}$ at $\ell=\ell_{0}$ are therefore expressed, respectively, as

$$
\frac{T(\ell, t)}{T_{0}}=\operatorname{erfc}\left[\frac{\theta \sqrt{D} B}{2 \sqrt{(t-\tau)}}\right], t>\tau ; \quad T(\ell, t)=0, t<\tau
$$

$$
\frac{T_{m}(\ell, z, t)}{T_{0}}=\operatorname{erfc}\left[\frac{\theta D B+(z-b)}{2 \sqrt{D(t-\tau)}}\right], t>\tau ; \quad T_{m}(\ell, t)=0, t<\tau
$$

where $D=\kappa / C_{m}$ is the rock thermal diffusion, $\theta$ is the ratio of rock to fluid heat capacity, i.e., $\theta=C_{m} / C$, while $\tau$ and $B$ represent the fluid advective travel time and the flow wetted surface (Moreno and Neretnieks, 1993), respectively,

$$
\tau=\int_{\ell_{0}}^{\ell} \frac{d \ell^{\prime}}{v\left(\ell^{\prime}\right)} ; \quad B=\int_{\ell_{0}}^{\ell} \frac{d \ell^{\prime}}{v\left(\ell^{\prime}\right) b\left(\ell^{\prime}\right)} .
$$

Note that for $t>>\tau$, the response of eq. (13) is controlled by the relationship between $t$ and the characteristic time $\theta^{2} D B^{2} / 4$. Solution of eq. (13) corresponds to the convolution over time of the response to an instantaneous pulse injection (Cvetkovic et al. (1999), Painter and Cvetkovic (2005)). In fact, given the linearity of the process, the superposition principle can be applied. In the case of uniform velocity and fracture aperture, eq. (13) coincides with the solution for a single fracture proposed by Tang et al. (1981).

\subsection{Single flow path with temperature-dependent fracture aperture and flow velocity}

We assume the same setting as in the previous section, consisting in a single trajectory defined by the longitudinal coordinate $\ell$, but with temperature-dependent fracture aperture and flow velocity, $b(\ell, t)$ and $v(\ell, t)$. The non-linearity of the problem hinders the derivation of a closed analytic solution. Considering a Lagrangian approach, we transform the Eulerian variables defined at $(\ell, t)$ into the Lagrangian variables defined at $\left[\tau(\ell) ; \ell_{0}, t_{0}\right]$, where $t_{0}=t-\tau$ and $\tau$ is the travel time to reach the position $\ell$, for a moving front originated at coordinate $\ell_{0}$ at time $t_{0}$, and displacing along the trajectory by advection, such as

$$
\tau=\int_{\ell_{0}}^{\ell} \frac{d \ell^{\prime}}{v\left[\tau(\ell) ; \ell_{0}, t_{0}\right]}
$$

All Lagrangian quantities are locally equal to their respective Eulerian quantities, such as

$$
\Upsilon\left[\tau(\ell) ; \ell_{0}, t_{0}\right]=\Upsilon\left(\ell, t_{0}+\tau\right)
$$

For simplicity we adopt the same nomenclature for the Eulerian and Lagrangian quantities, but we use a different notation to distinguish them, where the presence of the semicolon indicates the Lagrangian quantities. According to this framework, the energy balance in the fracture can be expressed as the variation of temperature of a moving front (Cvetkovic et al., 1999)

$$
\frac{\partial T\left[\tau(\ell) ; \ell_{0}, t_{0}\right]}{\partial t}+\frac{\partial T\left[\tau(\ell) ; \ell_{0}, t_{0}\right]}{\partial \tau}=-\frac{J\left(\ell, \tau+t_{0}\right)}{C b\left(\ell, \tau+t_{0}\right)},
$$

where we keep the terms in the right-hand side of the equation in terms of Eulerian quantities, ackwowleging the identity of eq. (17). The left-hand side of the equation can be rewritten in terms of total derivative $\mathrm{d} \bullet / \mathrm{d} \tau=\partial \bullet / \partial t+\partial \bullet / \partial \tau$, such as

$$
\frac{\mathrm{d}}{\mathrm{d} \tau} T\left[\tau(\ell) ; \ell_{0}, t_{0}\right]=-\frac{J\left(\ell, \tau+t_{0}\right)}{C b\left(\ell, \tau+t_{0}\right)} .
$$


Note that both the front temperature and velocity are not only a function of the position, and therefore of the travel time $\tau$, but they are also a function of the kick off time, $t_{0}$, and therefore of the global elapsed time $t=t_{0}+\tau$. The initial value of the front temperature, $T\left[0 ; \ell_{0}, t_{0}\right]$, constitutes a boundary condition of the problem. The temperature $T$ decays during the travel because of the diffusive heat exchange with the rock, $J$. This latter is defined in the Eulerian field and it is a function of the matrix temperature gradient at the fracture/matrix interface (eq. (4)). The temperature in the matrix, $T_{m}(\ell, z, t)$, is the result of the diffusion problem (recall Eq. (5)), whose linearity still holds. Solution of this latter for a variable BC allows us to obtain an expression for $J$ as the integral of the pulse heat transfer flux over the time (Appendix Appendix B)

$$
\begin{aligned}
J(\ell, t)= & -\kappa \int_{0}^{t} \frac{T_{m}\left(\ell, b, t^{\prime}\right)}{2 \sqrt{\pi D}\left(t-t^{\prime}\right)^{3 / 2}} \times \\
& \left.\exp \left(-\frac{(z-b)^{2}}{4 D\left(t-t^{\prime}\right)}\right)\left(1-\frac{(z-b)^{2}}{2 D\left(t-t^{\prime}\right)}\right) \mathrm{d} t^{\prime}\right|_{z=b} .
\end{aligned}
$$

The temperature at the fracture/rock interface, $T_{m}(\ell, b, t)$, is also defined in the Eulerian field and it is determined through continuity by the temperature of the front that is at that position at that specific time, such as

$$
T_{m}(\ell, b, t)=T(\ell, t)=T\left[\tau(\ell) ; \ell_{0}, t_{0}=t-\tau\right] .
$$

The formulation detailed so far acknowledges the variablein-time nature of the fracture aperture, which changes in response to the thermal deformation of the rock matrix. For example, the rock shrinks when it is cooled down, which causes an increase of the fracture aperture, while the rock expands when it is warmed up, which causes a decrease of the fracture aperture. Within the rock matrix, both the heat diffusion and the elastic deformation are linear problems, so superposition in time and space applies. Replacing $T_{m}(\ell, z, t)$ in eq. (11) by its expression in eq. (B.1) and solving the integral in $z$ we get

$$
\Delta b(\ell, t)=-\frac{1+v}{1-v} \alpha_{T} \sqrt{\frac{D}{\pi}} \int_{0}^{t} \frac{T_{m}\left(\ell, b, t^{\prime}\right)}{\sqrt{t-t^{\prime}}} \mathrm{d} t^{\prime},
$$

where we highlight that a negative temperature variation causes an increase in fracture aperture.

In the following we extend this approach to the case of multiple trajectories and we solve eqs. (19)-(22) by adopting a semianalytic scheme with time and space discretization.

\section{Formulation for multiple flow paths in heterogeneous de- formable fractured systems}

\subsection{Lagrangian fluid volume particles}

We now consider a three-dimensional heterogeneous fractured system. To solve the problem detailed in Section 2, we start by discretizing the duration of the injection in time steps $t_{0}$ with interval $\Delta t_{0}$.

We assume that the fracture aperture variation is slow, so as we consider the flow as quasi-steady state, i.e., steady state conditions applies during each time step with constant fracture aperture and velocity field. Under this assumption, we consider that the fluid flow is solved numerically by finite difference or finite element methods over a mesh grid. At each time step, the fracture aperture variation, and the consequent new fracture aperture, $2 b\left(\mathbf{x}, t_{0}\right)$, and velocity, $\mathbf{v}\left(\mathbf{x}, t_{0}\right)$, fields, are estimated. Once the velocity field in the fracture network is determined, it is possible to define multiple flow paths following the maximum pressure gradients and to simulate the advective transport by means of a Lagrangian particle tracking approach (Dagan and Bresler, 1979).

The condition of imposing a fixed temperature $T_{0}$ at the inlet corresponds to imposing the heat energy $E_{0}=C V_{0} T_{0}$ over a fluid volume $V_{0}$ entering the system for the duration of each time step, i.e., $V_{0}=Q_{0} \Delta t_{0}$, where $Q_{0}$ is the injected flow rate. We represent this energy as an ensemble of $N$ particles, which are released at each $t_{0}$ at locations $\mathbf{x}_{0}$ on the boundary. Particles are considered as fluid cells with equal volumes, whose sum equates the hot/cold volume introduced in the domain, $V_{0}$. The particle volumes do not undergo any change during the travel, i.e., any deformation keeps the volume unchanged (see top picture of Fig. 2). Although the conceptualization of particles as volume cells is useful for the purpose of this approach, the magnitude of the particle volume is irrelevant, as shown in the next section. Particles hold an energy $e$ and the total energy is given by the sum of the individual particle energy. Therefore, all particle volumes are initially charged with the energy $e_{0}=E_{0} / N$, and with the temperature equal to the injection temperature, $T\left(0 ; \mathbf{x}_{0_{q}}, t_{0}\right)=T_{0}, \forall q$. Note that we retain the same notation adopted in Section 3.2 to define particle quantities as a function of their residence time, injection position and injection time, e.g., $\Upsilon\left[\tau(\mathbf{x}) ; \mathbf{x}_{0}, t_{0}\right]$. Fluid particles change their energy because of their diffusive heat exchange with the rock matrix. Since we assume that both particle volume and heat capacity are constant, particle energy and particle temperature are related by a constant proportion. Therefore, we can arbitrarily adopt one or the other as the working variable. The adoption of the temperature will prove convenient to our purpose.

Particles move into the fracture system by advection along different trajectories that may change from a time step to another, due to the temporal variation of the velocity in the system. Each particle changes its position $\mathbf{x}$, such as

$$
\frac{\mathrm{d} \mathbf{x}\left(\tau ; \mathbf{x}_{0}, t_{0}\right)}{\mathrm{d} \tau}=\mathbf{v}\left(\tau ; \mathbf{x}_{0}, t_{0}\right), \quad \mathbf{x}\left(0 ; \mathbf{x}_{0}, t_{0}\right)=\mathbf{x}_{0}
$$

The particle Lagrangian velocity is equal to the local Eulerian velocity, e.g., $\mathbf{v}\left[\tau(\mathbf{x}) ; \mathbf{x}_{0}, t_{0}\right]=\mathbf{v}\left(\mathbf{x}, t_{0}\right)$, where we use the same notation for Lagrangian and Eulerian quantities as in the previous section, and where we apply eq. (17) highlighting that the velocity field is only updated at each time step. Similarly to Section 3.2, $\tau\left(\mathbf{x} ; \mathbf{x}_{0}, t_{0}\right)$ is the time that the particle, released at position $\mathbf{x}_{0}$ and time $t_{0}$, has spent to travel from $\mathbf{x}_{0}$ to the position $\mathbf{x}$. It can also be expressed as a function of the trajectory length, $\ell(\mathbf{x})$, and the particle velocity component in the direction parallel to the trajectory, $v_{\ell}\left[\ell(\mathbf{x}) ; \mathbf{x}_{0}, t_{0}\right]=\left|\mathbf{v}\left[\tau(\mathbf{x}) ; \mathbf{x}_{0}, t_{0}\right]\right|$, 
such as

$$
\tau\left(\mathbf{x} ; \mathbf{x}_{0}, t_{0}\right)=\int_{0}^{\tau} \mathrm{d} \tau^{\prime}=\int_{0}^{\ell(\mathbf{x})} \frac{\mathrm{d} \ell^{\prime}}{v_{\ell}\left[\ell^{\prime}(\mathbf{x}) ; \mathbf{x}_{0}, t_{0}\right]} .
$$

Thus, the particle arrival time at $\mathbf{x}$ is $t(\mathbf{x})=t_{0}+\tau\left(\mathbf{x} ; \mathbf{x}_{0}, t_{0}\right)$. Note that, in the case of constant in time fracture aperture, both the travel time and the velocity are not a function of $t_{0}$. Since space is discretized into a mesh grid, the particle travel time is calculated as the sum of the times to jump over the crossed mesh elements, where each jump is defined by the two successive intersections of the particle trajectory with the element edges.

In order acknowledge the variation of the velocity field, particles only move for the duration of the time interval $\Delta t_{0}$. If they have not reached the outlet during the time step in which they are injected, they resume their travel in the following time iteration along newly defined trajectories originating at their last position. This operation may imply that a large number of fractures are circulating within the network during each time step, which increases the computing memory demand. The adoption of a time interval larger than the advective time to reach the outlet prevent the simultaneous presence of multiple particle sets in the system, as we will further discuss in Section 8.

Particle injection in the network satisfies a flux-weighted injection mode and particle behavior at fracture intersections follows a complete mixing rule, i.e., particles are distributed proportional to the outgoing fluxes. This condition, together with the assumptions detailed above, implies the following relationship between the number of particles $n$, fluid volume $V_{e}$ and flow rate $Q_{e}$ entering a generic mesh element, and the number of particles $N$, fluid volume $V_{0}$ and flow rate $Q_{0}$ introduced in the system, for each time step

$$
\frac{n}{N}=\frac{V_{e}}{V_{0}}=\frac{Q_{e}}{Q_{0}} .
$$

\subsection{Particle temperature variation due to fluid-rock diffusion}

Particles change their temperature during the travel because of the thermal diffusion between the fluid and the rock matrix. The estimation of the particle temperature variation is performed by extending the semi-analytic method for a single trajectory detailed in Section 3.2 to the case of multiple trajectories. At each $t_{0}$ we release $N$ particles with assigned temperature at the inlet and we consider continuous injection during the time step. We differentiate the temperature at the fracturerock interface, $T_{m}(\mathbf{x}, b, t)$, defined in the Eulerian field, from the particle temperature, $T\left(\tau ; \mathbf{x}_{0}, t_{0}\right)$, defined in the Lagrangian field (Fig. 2). This dichotomy allows for defining the variation of $T\left(\tau ; \mathbf{x}_{0}, t_{0}\right)$ as a function of the diffusive heat flux (eq. (19)), which is estimated for each particle and it based on the local Eulerian temperature history, $T_{m}(\mathbf{x}, b, t)$ (eq. (20)). This latter is in turn determined by the temperature of the crossing particles, because of the equilibrium between fluid and rock temperatures at the interface (recall eq. (21)).

For a generic particle crossing the location $\mathbf{x}$ at time $t_{0}+\tau$, $T_{m}(\mathbf{x}, b, t)$ is equal to the temperature of the particle itself for the duration of the time interval, while for times smaller than $t_{0}+$

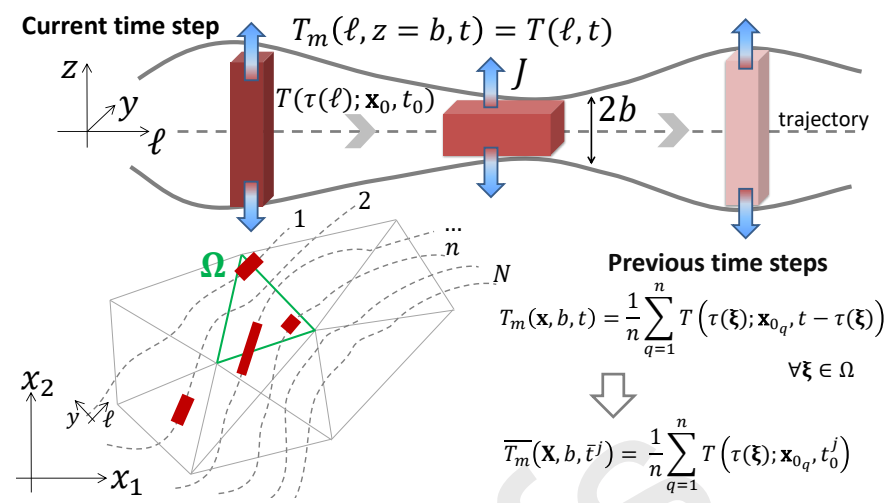

Figure 2: Schematic description of the particle-tracking approach for advective heat transport with fluid-rock diffusion and temperature-dependent fracture aperture and velocity. The top picture represents a single trajectory, the bottom picture shows the conceptual scheme for multiple trajectories.

$\tau$, it is equal to the temperature at the fracture/matrix interface collected at the grid mesh elements $T_{b}(\mathbf{X}, t)$, as expressed by

$$
T_{m}(\mathbf{x}, b, t)=\left\{\begin{array}{ll}
T\left[\tau(\mathbf{x}) ; \mathbf{x}_{0}, t_{0}\right], & t_{0}+\tau<t<t_{0}+\tau+\Delta t_{0} \\
T_{b}(\mathbf{X}, t), & t<t_{0}+\tau
\end{array},\right.
$$

where $\mathbf{X}$ represents the coordinate identifying the generic mesh element $\Omega(\mathbf{X})$ containing $\mathbf{x}$. This definition builds on the assumption that fluid particle volumes released at the same time do not directly exchange heat, because the differences in arrival times are small compared to the diffusive time to cross the distance between different trajectories. Although particle trajectories do not directly interact during their travel, particles have indirect effects on future particles. In fact, we consider that a time larger than the time interval is sufficient for a complete homogenization of each volume temperature to occur over the mesh element thanks to lateral diffusion processes. According with this assumption, the temperature at the mesh element, $T_{b}(\mathbf{X}, t)$, coincides with the temperature of the crossing fluid volume. Let $n\left(\mathbf{X}, t_{0}\right) \leq N$ be the number of particles released at the same $t_{0}$ that cross the mesh element $\Omega(\mathbf{X})$ at different times $t_{0}+\tau(\xi)$, where $\boldsymbol{\xi}$ is a generic position that falls within the mesh element $\Omega(\mathbf{X})$. It is worth reminding at this point that we assume continuous heat injection during the time step, which means that each temperature persists at each location for the duration of the time interval $\Delta t_{0}$. The total volume crossing $\Omega(\mathbf{X})$ in the time interval is $V$ and its temperature in time is determined by the sum of the energy of the particle volumes crossing the mesh element at that time, such that $T_{b}(\mathbf{X}, t)$ is

$$
\begin{aligned}
T_{b}(\mathbf{X}, t) & =\frac{1}{C V(\mathbf{X}, t)} \sum_{q=1}^{n\left(\mathbf{X}, t_{0}\right)} e\left[\tau(\boldsymbol{\xi}) ; \mathbf{x}_{0_{q}}, t-\tau(\boldsymbol{\xi})\right] \\
& =\frac{1}{n\left(\mathbf{X}, t_{0}\right)} \sum_{q=1}^{n\left(\mathbf{X}, t_{0}\right)} T\left[\tau(\boldsymbol{\xi}) ; \mathbf{x}_{0_{q}}, t-\tau(\boldsymbol{\xi})\right], \forall \boldsymbol{\xi} \in \Omega(\mathbf{X}) .
\end{aligned}
$$

The second equivalence is derived acknowledging that the 
energy of the generic particle $q$ is $e_{q}=C V_{q} T_{q}$ and that the total crossing volume $V$ is equal to the sum of the $n$ particle fluid volumes crossing the element, which is $V=n V_{q}$ because all the particles have equal and invariable volumes. By using the second equivalence, the estimation of $T_{b}(\mathbf{X}, t)$ does not depend on the magnitude of the particle volumes.

After substitution of eqs. (26) and (27) into eq. (20), and after integration by time intervals (see also Appendix Appendix B), the expression of the heat exchanged by a particle $p$ released at $t_{0}^{i}$, corresponding to the $i$-th time step, is

$$
J_{p}^{i}(\mathbf{x})=\sqrt{\frac{\kappa C_{m}}{\pi}} \frac{T_{p}^{i}(\mathbf{x})}{\Delta t_{0}}-J_{\Omega_{p}}^{i}(\mathbf{X}),
$$

where we make use of the generic short notation $\Upsilon_{p}^{i}(\mathbf{x})=$ $\Upsilon\left[\tau(\mathbf{x}) ; \mathbf{x}_{0_{p}}, t_{0}^{i}\right]$. The second term in the right hand side represents the memory diffusive heat exchange, which is defined on the mesh element such as

$$
\begin{gathered}
J_{\Omega_{p}}^{i}(\mathbf{X})=\sqrt{\frac{\kappa C_{m}}{\pi}} \sum_{j=1}^{i-1}\left[\frac{1}{n^{j}(\mathbf{X})} \sum_{q=1}^{n^{j}(\mathbf{X})} T_{q}^{j}(\boldsymbol{\xi}) \mathcal{F}\left[t_{p}^{i}(\mathbf{x}), t_{q}^{j}(\boldsymbol{\xi})\right],\right. \\
\forall \xi \in \Omega(\mathbf{X})
\end{gathered}
$$

where $t_{p}^{i}(\mathbf{x})=\tau_{p}^{i}(\mathbf{x})+t_{0}^{i}=\tau\left(\mathbf{x} ; \mathbf{x}_{0_{p}}, t_{0}^{i}\right)+t_{0}^{i}$, and where $f$ is defined as

$$
\mathcal{F}\left(t_{A}, t_{B}\right)=\frac{1}{\sqrt{t_{A}-t_{B}}}-\frac{1}{\sqrt{t_{A}-t_{B}+\Delta t_{0}}} .
$$

Expression (28) reflects the convolution over time of different temperature values, each one considered as constant over the time step. The first term represents the current temperature at the location $\mathbf{x}$, i.e., the temperature of the particle $p$ itself, while the second term represents the memory of all the past temperatures recorded at $\mathbf{x}$ over the time. The contributions of the different temperatures at the fracture/rock interface are superposed acknowledging the linearity of the diffusion process in the rock. Note that the denominators of eq. (30) are always real numbers as the terms in the square root are always positive. This is ensured by the fact that particles are stopped at the end of the time step, so the travel time to reach a given position is never larger than $\Delta t_{0}$.

It is worth reminding that the mutual effects between flow paths released in the same time step is disregarded, based on the assumption that differences in arrival time are small compared with the diffusive time. Acknowledging this effect would require sorting the particles by passage time order at each crossed element, which dramatically increases the computational cost. Despite that, the operation defined by eq. (29) still requires dealing with a large amount of data, which leads us to the simplification described in the next section.

\subsection{A meta-particle concept to reduce stored data}

At each time step and mesh element, the passage time and temperature of each particle have to be stored, which translates into huge sets of data considering that the particle number is also large. In order to reduce the amount of stored data, we build on the observation that the trajectories crossing a mesh element have similar temperatures and passage times. Therefore, at each time step we keep track of the effects of the particle passage time and temperature by assigning to each mesh element the values of a meta-particle, whose passage time and temperature is equal to the average passage times and temperatures of the $n$ particles released at that time step and crossing the element, such as

$$
\begin{gathered}
{\overline{T_{b}}}^{j}(\mathbf{X})=\frac{1}{n^{j}(\mathbf{X})} \sum_{q=1}^{n^{j}(\mathbf{X})} T_{q}^{j}(\boldsymbol{\xi}), \forall \boldsymbol{\xi} \in \Omega(\mathbf{X}), \\
\bar{t}^{j}(\mathbf{X})=t_{0}^{j}+\frac{1}{n^{j}(\mathbf{X})} \sum_{q=1}^{n^{j}(\mathbf{X})} \tau_{q}^{j}(\boldsymbol{\xi}), \forall \boldsymbol{\xi} \in \Omega(\mathbf{X}),
\end{gathered}
$$

where the meta-particle variables are expressed using again the short notation, e.g., $\bar{\Upsilon}^{j}(\mathbf{X})=\bar{\Upsilon}\left[\tau(\mathbf{X}) ; \mathbf{x}_{0}, t_{0}^{j}\right]$. Note that, since the discretization for $\boldsymbol{\xi}$ corresponds to the mesh edges, i.e., the intersections between the particle trajectories and the mesh elements, we estimate $\bar{T}_{b}^{j}(\mathbf{X})$ and $\bar{t}^{j}(\mathbf{X})$ as the average between the values at the two adjacent edges of $\Omega(\mathbf{X})$.

These average times and temperatures substitute $T_{b}$ in eq. (26), such as $T_{b}(\mathbf{X}, \bar{t}(\mathbf{X}))=\overline{T_{b}}(\mathbf{X})$. Under this framework, the heat flux that a particle $p$ released at time $t_{0}$ exchanges at the position $\mathbf{x}$ is still expressed by eq. (28), but the memory diffusive heat exchange is evaluated according with the meta-particle temperatures and passage times recorded at the position over the time steps, such as $J_{\Omega_{p}}^{i}(\mathbf{X})$ is substituted by $\overline{J_{\Omega_{p}}} i(\mathbf{X})$

$$
{\overline{J_{\Omega_{p}}}}^{i}(\mathbf{X})=\sqrt{\frac{\kappa C_{m}}{\pi}} \sum_{j=1}^{i-1}{\overline{T_{b}}}^{j}(\mathbf{X}) \mathcal{F}\left[t_{p}^{i}(\mathbf{x}), \bar{t}^{j}(\mathbf{X})\right] .
$$

The adoption of this meta-particle concept sensibly reduces the amount of stored data, with respect to evaluating the diffusive flux considering the individual contribution of all the crossing particles released in the previous time steps, as described in the previous section. At the end of each time step, the recorded particle passage times and temperatures of each location are translated into one value of temperature and associated time for each fracture mesh element. The consequent loss of accuracy is minimal, as we discuss in Section 5.4.

Thus, the particle temperature variation during the advective displacement across the element $\Omega\left(\mathbf{X}_{k}\right)$ is evaluated by solving eq. (19), which is discretized according to a Crank-Nicolson scheme into

$$
\frac{T_{p}^{i}\left(\mathbf{x}_{k^{+}}\right)-T_{p}^{i}\left(\mathbf{x}_{k^{-}}\right)}{\tau_{p}^{i}\left(\mathbf{x}_{k^{+}}\right)-\tau_{p}^{i}\left(\mathbf{x}_{k^{-}}\right)}=-\frac{J_{p}^{i}\left(\mathbf{x}_{k^{+}}\right)-J_{p}^{i}\left(\mathbf{x}_{k^{-}}\right)+2{\overline{J_{\Omega_{p}}}}^{i}\left(\mathbf{X}_{k}\right)}{2 C b\left(\mathbf{X}_{k}, t_{0}^{i}\right)}
$$

where the subscripts $k^{+}$and $k^{-}$identify the trajectory entrance and exit points in the grid element $\Omega\left(\mathbf{X}_{k}\right)$. Note that the fracture half-aperture value refers to the injection time. In fact, the fracture aperture and velocity fields are updated at each time step and they are considered constant for the duration of the time step. The fracture half-aperture variation is estimated at each 
mesh element according to the meta-particle average values of temperature and time, such that eq. (22) translates into

$$
\Delta b^{i}(\mathbf{X})=2 \frac{1+v}{1-v} \alpha_{T} \sqrt{\frac{D}{\pi}} \sum_{j=1}^{i} \bar{T}_{b}^{j}(\mathbf{X}) \mathcal{G}\left[\bar{t}^{i}(\mathbf{X}), \bar{t}^{j}(\mathbf{X})\right],
$$

where

$$
\mathcal{G}\left(t_{A}, t_{B}\right)=\sqrt{t_{A}-t_{B}}-\sqrt{t_{A}-t_{B}+\Delta t_{0}} .
$$

Finally, the fracture semi-aperture field is updated at each time step according to the more recently recorded values of $\Delta b$ at each $\mathbf{X}$, such as

$$
b^{i+1}(\mathbf{X})=b^{0}(\mathbf{X})+\Delta b^{i}(\mathbf{X}) .
$$

Note that this formulation disregards the effects of lateral mechanical continuity because the thermal displacement is assumed as exclusively dependent on the temperature variation in the direction perpendicular to the fracture, while in practice mechanical deformation is instantaneously transferred far from the thermal perturbation through the solid matrix. In other words, the fracture opening should propagate laterally both in the fracture longitudinal, $\ell$ and on-plane transverse, $y$, directions. However, the lack of this effect is partly compensated by the smooth nature of the heat propagation in the fracture, both in the longitudinal direction, as a consequence of the diffusive exchange with the matrix, and in the on-plane transverse direction, because of the similar velocities of adjacent streamlines over the same fracture. We test the validity of this assumption in Section 5.3 .

\subsection{Implementation in a DFN simulator}

The formulation has been implemented in the DFN.lab software platform, which provides a set of tools for the generation of stochastic and deterministic heterogeneous fracture networks, and for the modeling of flow in the connected network through mixed hybrid finite element scheme (Le Goc et al., 2019; Pinier et al., 2021). This scheme ensures fluid mass conservation, including for unstructured meshes with a high variability in element quality. The particle tracking module, already implemented in the software, constitutes the basis for the advective part of this formulation. The algorithm is designed to develop accurate particle tracing on unstructured meshes (Matringe et al., 2006).

Particle trajectories are defined on the grid mesh according with the velocity field, which is approximated as constant within each element. At each element crossed, the particle motion occurs on a straight line. The velocity vector determines the exit point, i.e., the position where the trajectory crosses the element edge, while the velocity norm determines the time for the particle to jump across the element. Since particle motion occurs for the duration of the time interval (Section 4.1), it may occur that some particles have not reached the outlet during the time interval. In this case, particles are stopped and their travel resume when a new time-step is initiated. To do so, the particle position at the stop time is evaluated by means of a linear interpolation on the trajectory line crossing the last visited element.

\section{Validation of the proposed approach}

\subsection{Validation and convergence analysis in the case of con- stant fracture aperture and velocity}

In the case of a single trajectory with constant in time fracture aperture and velocity, the semi-analytic solution described in Sections 3.2 and 4 should provide the same estimates of temperature than the analytic solution of Section 3.1. We make use of this equivalence to analyze the convergence performance of the semi-analytic solution under different conditions of time discretization. We consider a fracture of $10 \mathrm{~m}$ length with constant in time fracture aperture and steady state flow velocity. This corresponds to either constant flow or constant pressure boundary conditions at the fracture inlet $x=0$, where we also impose a constant temperature condition. The rock matrix thermal diffusivity is $D=1 \times 10^{-6} \mathrm{~m}^{2} / \mathrm{s}$ and the parameter $\theta$ is 1 , i.e., $C_{m}=C$.

We test the performance of the semi-analytic formulation for two cases, one with uniform velocity and regular spatial grid, the other with more general conditions of non-uniform velocity and irregular spatial grid. For both cases the semi-aperture is uniform and equal to $b=1 \times 10^{-4} \mathrm{~m}$. For the first case, we consider flow velocity $v=2.5 \times 10^{-2} \mathrm{~m} / \mathrm{s}$. We adopt a space discretization such that $\Delta x=1 \mathrm{~m}$, while for the time discretization we consider different time intervals $\Delta t_{0}$. To keep generality, the analysis is performed in terms of dimensionless values making use of the characteristic time $t_{c}=\theta^{2} D \Delta \tau^{2} /\left(\pi b^{2}\right)$, which represents the ratio of the square of the advective characteristic time for the single jump, $\Delta \tau=\Delta x / u$, to the diffusive characteristic time, $\pi b^{2} /\left(\theta^{2} D\right)$. The value of the characteristic time is approximately 0.5 days for this specific example. The adoption of $t_{c}$ will prove convenient, as shown in the following.

Figure 3 shows the comparison between the two solutions under values of time intervals from $0.1 t_{c}$ to $10 t_{c}$, which correspond to values from $10^{2} \Delta \tau$ to $10^{4} \Delta \tau$. In the left panel, the temperature variation in time is represented for three different control planes, at $x=1.5 \Delta x, x=3.5 \Delta x$ and $x=9.5 \Delta x$, respectively. Since values are stored on the grid element, we take the middle point of the element as $x$. At the first control plane, the temperature starts to change for time larger than $0.2 t_{c}$, which is expected as in eq. (13) the temperature is larger than zero for arguments of the complementary error function smaller than 2 , i.e., when $t>t_{c} / 5$ if $t>>\tau$. In the right panel, we show the result vs space at $t=30 t_{c}$. The semi-analytic solution shows a good agreement with the classical solution and it is most accurate for smaller values of $\Delta t_{0} / t_{c}$. The error increases for increasing values of the time interval and it incurs an overestimation of the temperature, especially for small times. In fact, if eq. (28) is replaced into eq. (34), one obtains that the temperature variation of the front depends on the ratio $\theta \sqrt{D} \Delta \tau / \sqrt{\pi \Delta t_{0}} / b$, which coincides with $\sqrt{t_{c} / \Delta t_{0}}$. Overall, we observe that the adoption of the Crank-Nicolson scheme ensures stability, while preserving accuracy.

We also perform a convergence analysis for the case of nonuniform velocity and non-uniform space discretization (Fig. 4). The fracture semi-aperture is assumed as uniform and equal to 

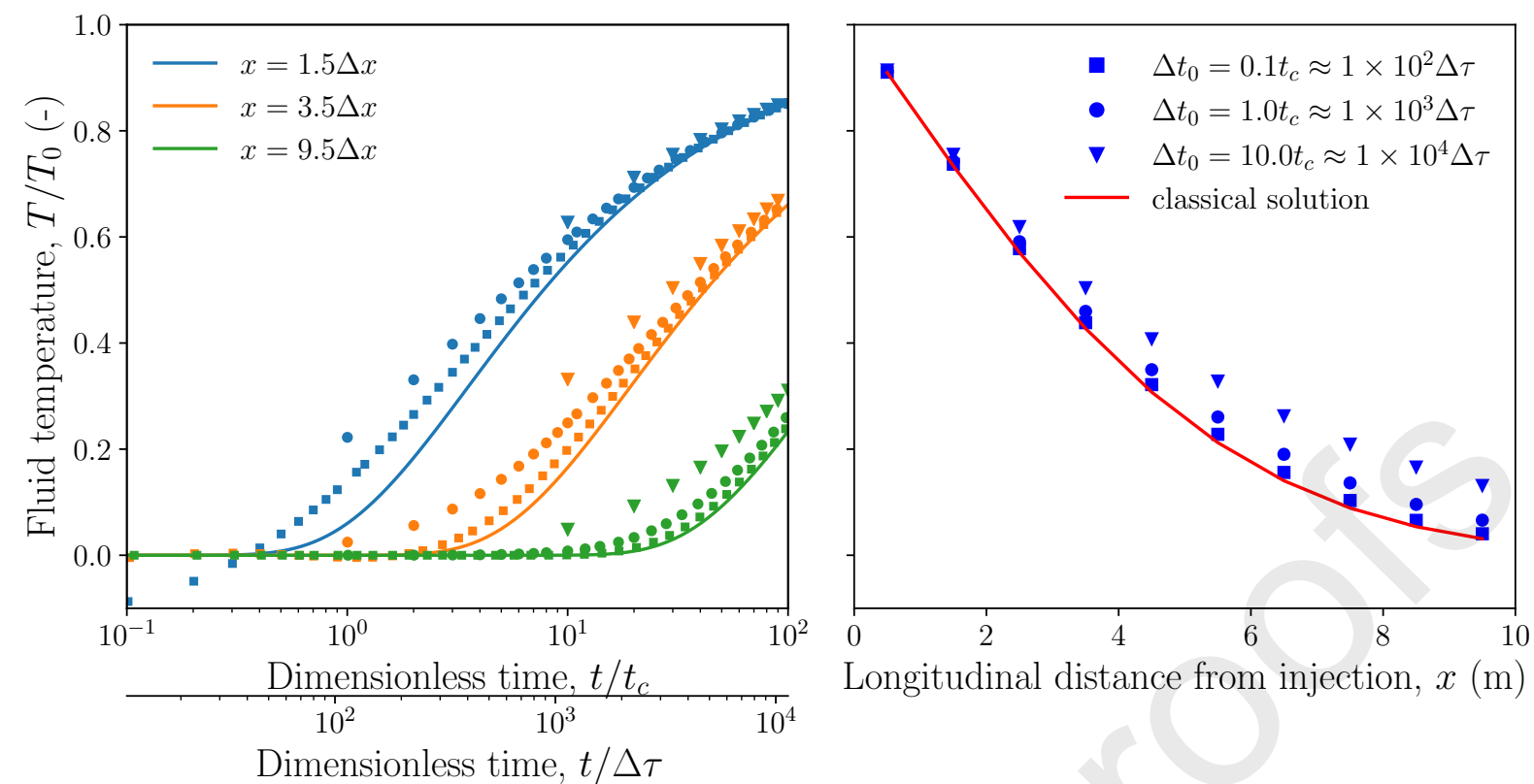

Figure 3: Temporal (left) and spatial (right) evolution of temperature in the fracture in response to non-isothermal continuous injection for the case of constant in time and uniform fracture aperture and velocity. Comparison of the results calculated by means of the proposed semi-analytic approach (markers), as described in Sections 3.2 and 4, and by the classical solution, as in eq.(13) (solid lines). The different colors in the left panel correspond to different control planes along the fracture trajectory, which is discretized with space interval $\Delta x=1 \mathrm{~m}$. The different markers refer to different values of time intervals for the semi-analytic solution. Time is normalized with respect to the characteristic time $t_{c}=\theta^{2} D \Delta \tau^{2} / \pi / b^{2}$, where $\Delta \tau$ is the advective time for the single jump $\Delta \tau=\Delta x / u$. Right panel shows results for $t=30 t_{c}$. Plot-frequency is adjusted to improve visualization.

$b=1 \times 10^{-4} \mathrm{~m}$, which means that the variation of velocity corresponds to an equivalent variation of fluid flow. With this example, we mimic the general case of a trajectory crossing several fractures and intersections, like in a complex fracture network. Although we keep the fracture aperture as uniform, the variability of the advective time for each jump is such that the characteristic time $t_{c}$, evaluated for each space grid element, spans over several orders of magnitude and it is distributed as shown in the inset of Fig. 4. We test the convergence assuming values of time interval proportional to the average characteristic time $\overline{t_{c}}$. The results confirm the accuracy of the solution under a strong heterogeneity, although some oscillations are observed at short times for the case of small time interval.

Although results are sufficiently accurate for all the time intervals, a small time interval may incur large computational time, while if the time interval is very large, temperatures are not calculated for short time, i.e., time smaller than the time interval. Considering the large variability of the characteristic time in a heterogeneous medium, the evaluation of a minimum and maximum time interval at the beginning of the simulation may be useful to prevent from this inconvenient.

\subsection{Convergence analysis in the case of variable fracture aper- ture and velocity}

Here we analyze the consequences of the thermo-mechanical coupling for a single fracture. We compare the heat transfer for the case of constant fracture aperture and velocity with the case when the fracture aperture and the flow velocity change because of the temperature-dependent deformation.
We consider the same setting and parameter values as for the first case of Section 5.1, with initial uniform semi-aperture $b^{0}$ equal to $1 \times 10^{-4} \mathrm{~m}$ and uniform space discretization $\Delta x=1$ $\mathrm{m}$. However, to evaluate the velocity field variation consequent to the fracture aperture variation, we distinguish two different cases of boundary conditions (BCs) at the inlet. In one case we consider constant inflow, in the other we assume constant pressure. In both cases, the outlet at $x=L$ is subject to a constant zero pressure BC and the initial velocity is $v^{0}=2.5 \times 10^{-2} \mathrm{~m} / \mathrm{s}$. Since we consider the case of a single fracture with no intersections, the condition of steady state flow at each time step implies that the flow is uniform along the trajectory. Although we show the results by means of dimensionless parameters, the aperture variation depends on the injected temperature, which we fix as $T_{0}=-10^{\circ} \mathrm{C}$. For the mechanical parameters, the linear thermal expansion $\alpha_{T}$ is $1 \times 10^{-5}\left({ }^{\circ} C\right)^{-1}$ while the Poisson ratio $v$ is 0.3 . We consider three different time interval values, from $0.1 t_{c}^{0}$ to $10 t_{c}^{0}$ in order to show the convergence performance under the circumstance of changing aperture. The characteristic time $t_{c}^{0}$ refers to the initial values of semi-aperture, $b^{0}$, and velocity, $v^{0}$. Therefore, it has the same value as in the Section 5.1, i.e., $t_{c}^{0} \approx 0.5$ days.

Figure 5 shows the temporal variation of temperature and fracture semi-aperture, observed at one control plane placed at $x=3.5 \mathrm{~m}$ from the inlet, for the case with constant aperture and variable aperture under the two different BCs. In the case analyzed here, the cooling of the rock causes an increase in fracture aperture. This implies the variation of both the flow velocity and the fluid volume exchanging heat with the rock. This latter becomes larger, which leads to smaller temperature 

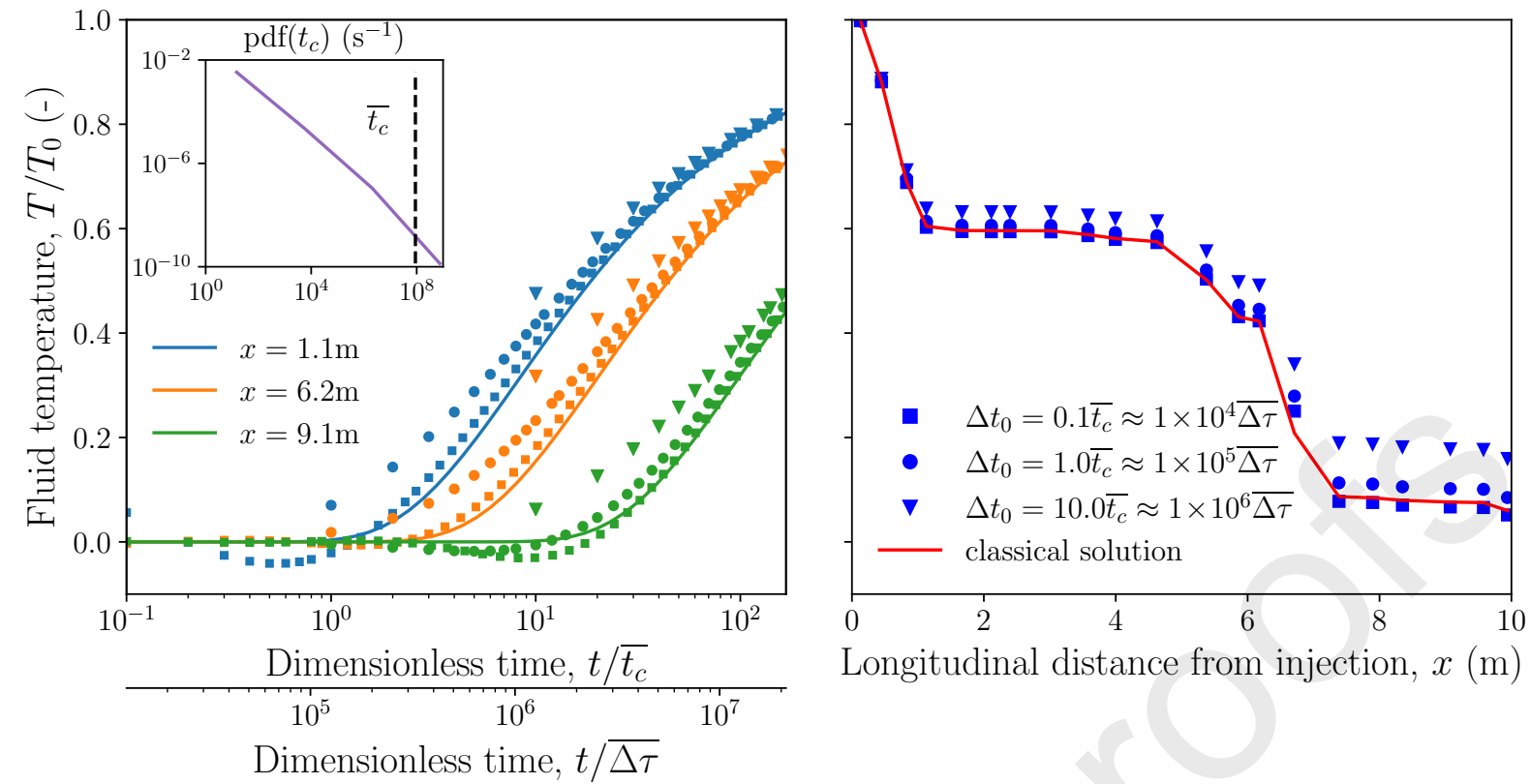

Figure 4: Temporal (left) and spatial (right) evolution of temperature in the fracture in response to non-isothermal continuous injection for the case of constant in time but not uniform velocity, and with non-uniform spatial discretization. The variability of the characteristic time, evaluated for each mesh element, is shown in the inset plot of the left panel. Comparison of the results calculated by means of the proposed semi-analytic approach (markers), as described in Sections 3.2 and 4 , and by the classical solution, as in eq. (13) (solid lines). The different colors in the left panel correspond to different control planes along the fracture. The different markers refer to different values of time interval for the semi-analytic solution. Time is normalized with respect to the average characteristic time, represented in the inset plot by the black dashed line, and by the average advective time. Right panel shows results for $t=30 \overline{t_{c}}$. Plot-frequency is adjusted to improve visualization.

variations for unitary advective displacement. Note that in the case of constant aperture, the velocity is invariant regardless of the $\mathrm{BC}$. When the aperture is temperature-dependent, on the contrary, the $\mathrm{BC}$ has a great impact on the velocity, which is also shown in Fig. 6, where results are presented as a function of the longitudinal direction, for the sole case with time interval equal to $t_{c}^{0}$. If the inflow is kept constant at the inlet, flow velocity changes proportionally to the inverse of the local fracture half-aperture, i.e., $v(x, t) \propto b(x, t)^{-1}$. This implies that the advective time increases proportionally to the increase of $b$, which counterbalances the increase of fluid volume in contact with the rock. Therefore, the characteristic time remains equal to the initial conditions, and to the constant aperture case. This has two consequences. The first is that the convergence is not affected. Second, the temperature response remains the same as in the constant case. This equivalence of results is specific of considering a single trajectory in a one-dimensional domain. With two-dimensional fractures in a three-dimensional domain, the variation in velocity promotes the migration of the trajectory towards faster preferential zones, as we will see in Section 7.

The behavior is different when a constant pressure is imposed at the inlet. In this case, flow velocity is proportional to $[b(x, t) I(t)]^{-1}$, where $I(t)=\int_{0}^{L} b(x, t)^{-3} d x$, according with the assumption that the cubic law applies locally (see Section 2.1). The characteristic time is therefore proportional to the square of $I(t)$ for any $x$. This non-locality affects both the observed response and the convergence conditions. At short times, the increase in fracture aperture only occurs over a small portion of the domain (observe Fig. 6). The velocity is reduced for short $x$ and slightly increased for large $x$. The value of the characteristic time is similar to the initial one, which makes the temperature responses for constant and variable aperture comparable. Later on, the aperture variation propagates to the rest of the domain, which gives a major importance to the integral $I$, compared to $b$. The consequent acceleration of the temperature front in turn increases the propagation of the aperture variation, which dramatically speeds up the heat transfer, giving the abrupt evolution of temperature observed in Fig. 5.

As said, when a constant pressure BC is imposed, the characteristic time of the problem changes according to the square of the space integral $I$, which has direct consequences on the accuracy of the calculation. If the aperture increases, corresponding to a cooling of the rock, the characteristic time decreases, which means that the relationship $\Delta t_{0} / t_{c}$ increases. This may generate less accurate results and lack of information (Fig. 5). On the contrary, if the rock is heated up, the consequent reduction of $b$ ensures that the relationship $\Delta t_{0} / t_{c}$ decreases, and therefore that the accuracy is preserved, as shown in Supplementary Information.

\subsection{Validation of the thermo-mechanical response}

The accuracy of the analytic evaluation of the TM fracture aperture (eqs. (22) and (35)) is tested against results from a fully coupled THM numerical simulation performed with the Finite Element Method simulator CodeBright (Olivella et al., 1996). We consider a single fracture embedded in a rock matrix. Both the fracture and the rock are represented as porous media in the finite element simulator. Therefore, we build the 

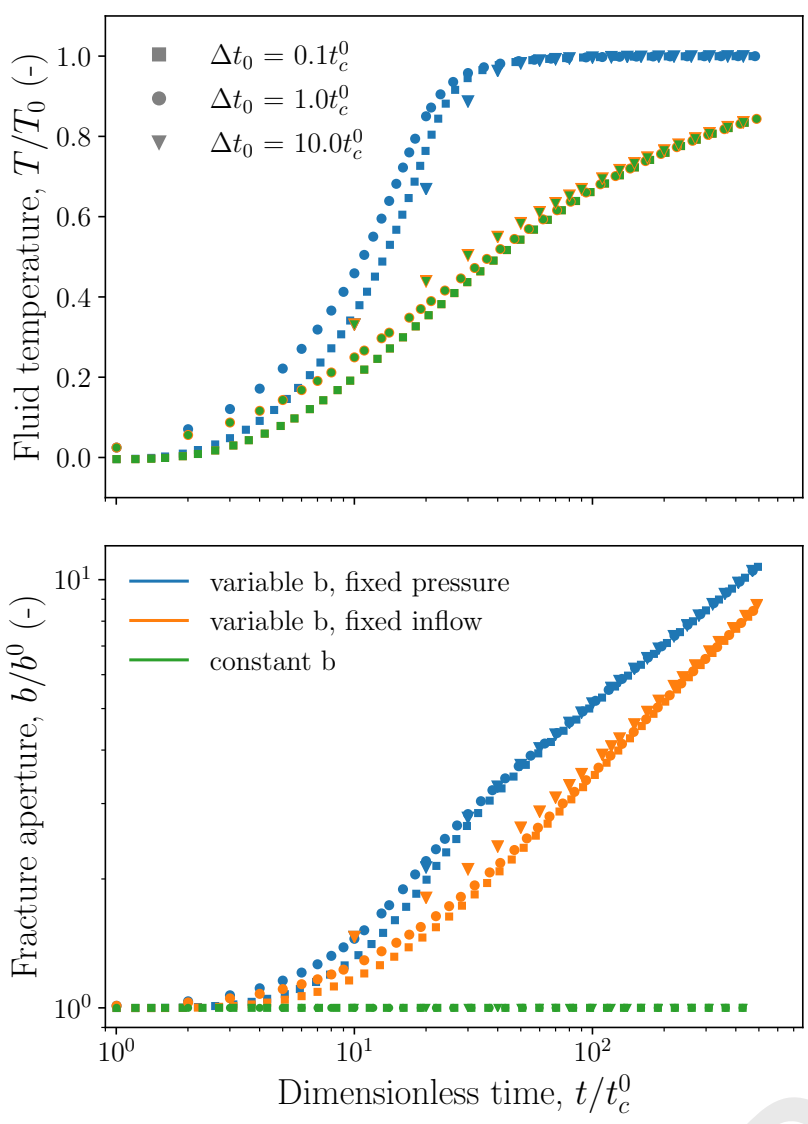

Figure 5: Temporal evolution of fluid temperature (top) and semi-aperture (bottom) for a fracture subject to cold fluid injection. Results correspond to a control plane placed at $3.5 \mathrm{~m}$ from the injection section at $x=0$. The case with constant fracture aperture and velocity (green) is compared with the case of temperature dependent fracture aperture and velocity, under the inlet $\mathrm{BC}$ of constant pressure (blue) or constant inflow (orange). The different markers correspond to different values of the time interval, from 0.1 to 10 times the initial characteristic time $t_{c}^{0}$, as defined in the text. Fluid temperature is normalized with respect to the temperature injected at the inlet, semi-aperture is normalized with respect to its initial value, while time is normalized with respect to $t_{c}^{0}$. Marker plot-frequency is adjusted to improve visualization.

model in order to have it consistent with our assumptions. We set the longitudinal thermal conductivity in the fracture as very low, in order to the heat transport in the fracture be governed by advection, according with the assumptions here. A low intrinsic permeability is assigned to the rock matrix in order to make it consistent with our assumption that the rock is impermeable and that the heat transport in the rock is exclusively driven by thermal diffusion. We impose a small pressure gradient with a highly conductive fracture, to reduce the hydro-mechanical deformations and only observe the thermo-mechanical deformations. The viscosity is set as insensitive to the temperature variations. We assume constant velocity, i.e., the fracture aperture variation does not affect the velocity field or the transport behavior. Thus, we only evaluate the aperture variation in response to the temperature field generated by a continuous injection for a certain time. The fracture is $100 \mathrm{~m}$ long with a steady state flow rate of $5 \times 10^{-5} \mathrm{~m}^{2} / \mathrm{s}$. The rock thermal diffusivity $D$
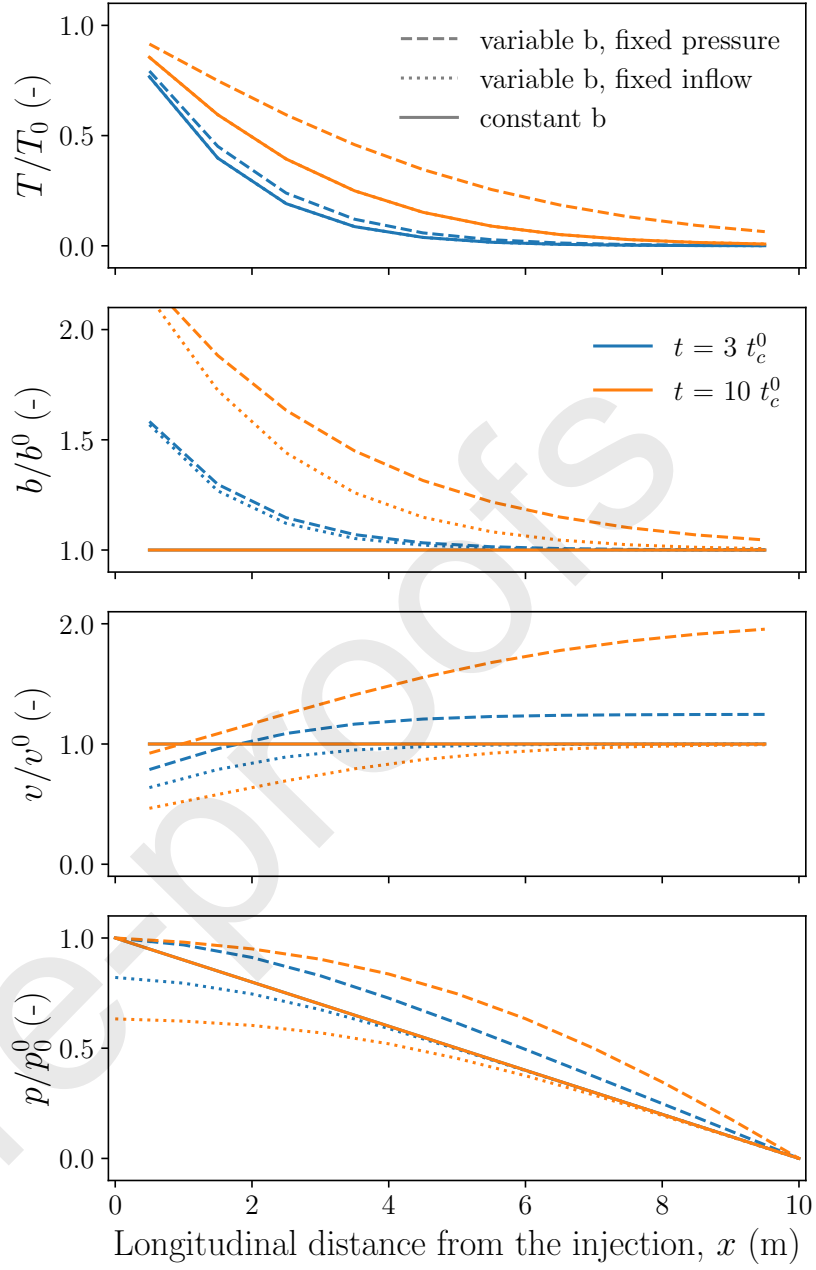

Figure 6: From top to bottom: variation of fluid temperature $T$, fracture semiaperture $b$, flow velocity $v$, and pressure $p$ as a function of the distance from the injection section for a fracture subject to cold fluid injection. Results refer to two different observation times at dimensionless time equal to 3 (blue lines) and 10 (orange lines) with respect to the initial characteristic time $t_{c}^{0}$. The case with constant fracture aperture and velocity (solid lines) is compared with the case of temperature-dependent fracture aperture and velocity, under the case of constant pressure BC (dashed lines) or constant inflow BC (dotted lines) at the inlet. $T$ is normalized with respect to the temperature injected at the inlet, $b$ and $v$ are normalized with respect to their initial values, $p$ with respect to the initial pressure at the inlet, while time is normalized with respect to $t_{c}^{0}$. Estimations are performed by adopting $\Delta t_{0}=t_{c}$.

is equal to $1.6 \times 10^{-6} \mathrm{~m}^{2} / \mathrm{s}$ and the parameter $\theta$ is approximately 0.9 . We simulate 20 days of continuous injection of the temperature $T_{0}$ at the $x=0$. Nevertheless, we show the results in terms of dimensionless time, which allows for extending them to other sets of parameters. To this end, the characteristic time $t_{c}=\theta^{2} D \tau^{2} / \pi / b^{2}$ is adopted, as described in the previous section, where the characteristic length for $\Delta \tau$ is set as equal to 1 $\mathrm{m}$. For the mechanical parameters, the linear thermal expansion $\alpha_{T}$ is $1 \times 10^{-5}\left({ }^{\circ} \mathrm{C}\right)^{-1}$ while the Poisson ratio $v$ is 0.3 . We set the domain size such that the mechanical boundary conditions do not affect the behavior.

Figure 7 shows that the analytic estimation of both the temperature and the semi-aperture variation is very accurate, which 
confirms the validity of the assumptions described in Section 2.3. We observe that considering the mechanical problem as one-dimensional in $z$ does not incur large errors, because the lateral mechanical propagation of the displacement is reproduced by the smooth heat propagation. Small discrepancies are observed when the fluid temperature profile is sharp (observe the blue and orange lines), a condition that is slightly smoothed out by the fully coupled numerical simulator. This impacts the rock temperature profiles, and consequently the strains, for short times.

\subsection{Accuracy of the meta-particle assumption}

As described in Section 4.3, the memory diffusive heat exchange is calculated by making use of a meta-particle concept, i.e., at each time step we operate an averaging of the arrival time and temperature over the particles crossing each grid mesh element. Here we test the effects of this averaging by comparing the particle-rock heat exchange flux and the particle temperatures after the diffusive exchange, evaluated considering the meta-particle (eq. (33)) or the individual particle temperatures (eq. (29)).

To perform this analysis, we consider observing one location at which 10 particles arrive with a certain distribution of passage time and temperature. We evaluate the fluid-rock heat exchange for each particle, $J_{p}$, and the consequent outgoing particle temperature after having exchanged $J_{p}$ for a $\Delta \tau=1 \mathrm{~s}$. This is performed for several observation times, corresponding to the passage of particle sets released at different $t_{0}$. The parameters $b, \theta$ and $D$ are set as in Section 5.1. The characteristic time for the unitary $\Delta \tau$ is therefore equal to $50 \mathrm{~s}$. Without loss of generality, we consider stationary trajectories, i.e., the particle sets released at different $t_{0}$ cross the control location with constantin-time values of passage time $\tau$. Their probability density function (pdf) follows an upper truncated power law distribution with exponent $\gamma$, such as $\operatorname{pdf}(\tau)=\gamma \tau^{-\gamma-1} /\left(\tau_{m}^{-\gamma}-\tau_{M}^{-\gamma}\right)$. The minimum and maximum cutoff values, $\tau_{m}$ and $\tau_{M}$, are set as $10 \mathrm{~s}$ and 1010s, respectively. We explore two scenarios with values of $\gamma$ equal to 0.5 and 2.5. For the time interval of particle release $\Delta t_{0}$, we consider different values corresponding to ratios of $\left(\tau_{M}-\tau_{m}\right)$ to $\Delta t_{0}$ ranging between 0.1 and 1. Each particle crosses the control location with a temperature correlated with its passage time and with the observation time by means of the relation $T \propto \operatorname{erfc}(\tau / \sqrt{t})$, according with eq. (13), where we assume that the imposed initial temperature is $T_{0}=50^{\circ} \mathrm{C}$. This assumption reflects the condition in which the particles have traveled through independent trajectories before converging to the control location. Therefore particle temperature increases with increasing observation time and with decreasing passage time, i.e., fast particles have higher temperature than slow ones. Moreover, the variability is large for short observation time, and it reduces as the observation time increases. For each scenario, we perform 100 realizations and we evaluate the average of the maximum differences in particle exit temperature.

Figure $8 \mathrm{a}$ and $\mathrm{b}$ show results at different observation times for one realization of the case with $\gamma=0.5$ and $\Delta t_{0}=\tau_{M}-\tau_{m}$. To improve visualization, results are plotted with a frequency time smaller than $\Delta t_{0}$. Panel a shows the diffusive heat exchanged by each particle, $J_{p}$, at the observed location and at different times when the contribution of all particles (blue markers) or the meta-particle contribution (orange markers) is considered. At each observation time, the particles cross the location with temperature proportional with their passage time. The heat that a particle exchanges depends on its temperature and on the local rock temperature. Therefore, $J_{p}$ is larger for the first arrival particles, which have a higher temperature (observe the inset plot). Hottest particles undergo a positive heat exchange (they give heat), while coldest particles undergo a negative heat exchange (they receive heat). In both cases, the absolute values of $J_{p}$ first increase with time and eventually decrease as larger rock volumes are heated up. This time of trend inversion depends on the particle passage times and temperatures. For the example analyzed here, the inversion time is larger than the simulated time for the slowest particles, so we do not observe any reduction of $J_{p}$ for those particles. Note also the sign variation of $J_{p}$ for particles with intermediate velocity, which indicates that they receive heat at the beginning, while they give heat later. Results show that considering all the values of particle temperature and passage time or the meta-particle average values gives similar results (compare the blue and orange markers).

After having exchanged $J_{p}$, the particles exit the location with a new temperature. Figure $8 \mathrm{~b}$ shows the relative difference between the outgoing temperatures evaluated with the metaparticle values and considering the particle contributions with their respective $\tau$ and $T$. Values are normalized with respect to the latter. After the first observation time, when the two methods give the same outgoing temperatures, they diverge. In particular, the error is positive, but small, when the meta-particle method is adopted, and it reduces with time.

The trend is similar when other scenarios are analyzed, for which the maximum positive and negative errors, averaged over the 100 realizations, are shown in Fig. 8c. Results are shown for the two scenarios of $\gamma$, with each line of the plot corresponding to a different value of the time interval $\Delta t_{0}$ and columns representing different observation times. The error associated with the meta-particle averaging operation is always positive (the maximum negative colorplot exhibits zero values). The accuracy of the averaging operation is sensitive to the ratio of $\left(\tau_{M}-\tau_{m}\right)$ to $\Delta t_{0}$, which we explore by varying $\Delta t_{0}$. The error increases when the ratio increases, reflecting that the averaging process incurs overestimation of the particle temperature if the time interval is similar to the maximum difference in particle arrival time. This occurs under all the assumed scenarios, but it is particularly evident for smaller $\gamma$, i.e., when the number of particles with large arrival time is larger. Moreover, the error is larger for short times and decreases as the time increases, which is a consequence of the reduction of the variability in particle arrival temperature with time. In general, large variability increases the error, yet the maximum error is overall smaller than $5 \%$, which confirms the accuracy of the meta-particle method.

Although we have performed the analysis assuming the case of a hot fluid injection, the same normalized results would be found in the case of cold injection. 

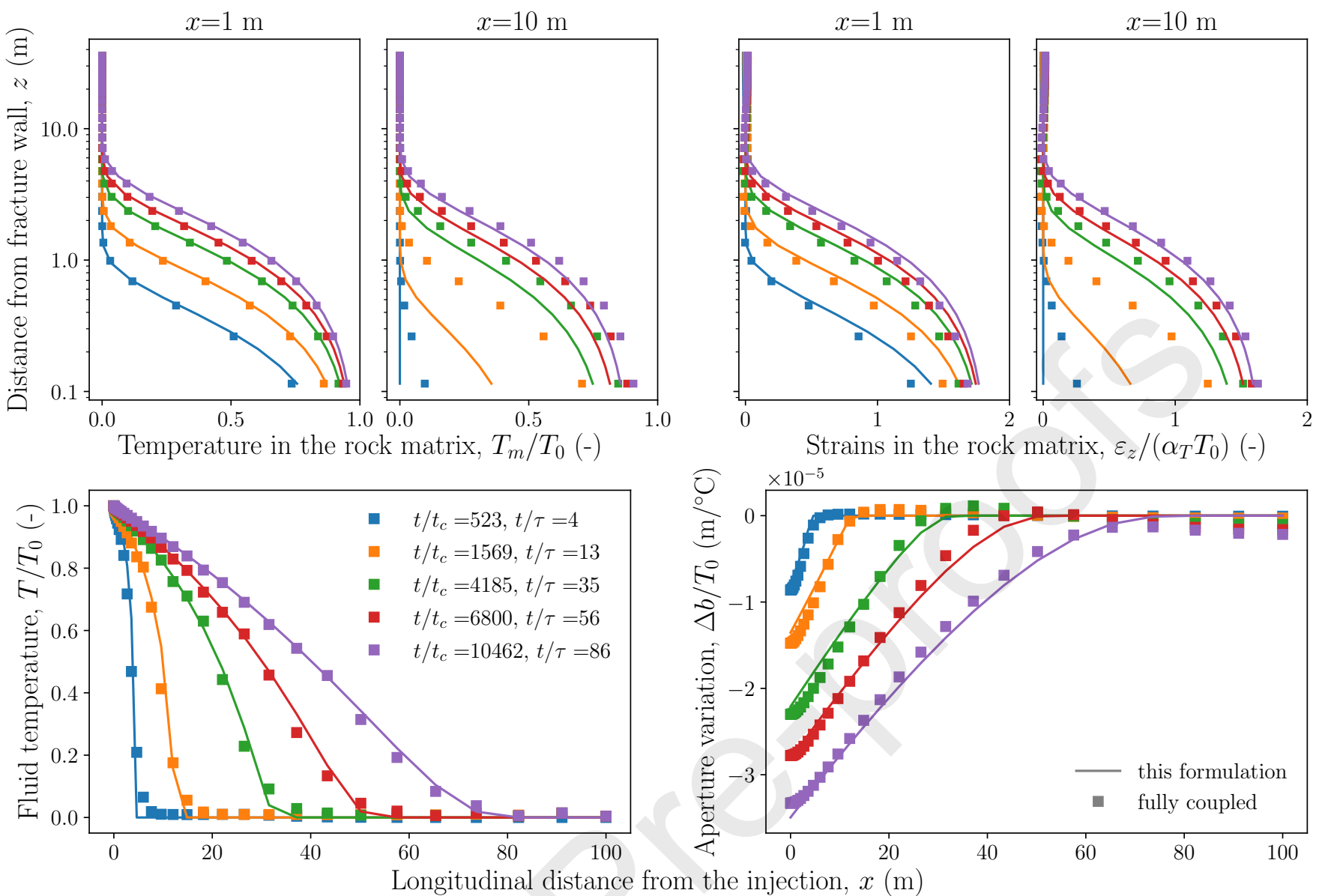

Figure 7: Comparison of the thermo-mechanical behavior of a fracture subject to continuous injection evaluated by means of the analytic solution described in Sections 3.2 and 4 (lines) and by a fully coupled thermo-hydro-mechanical numerical simulation (markers). Top panels show the evolution of temperature (left panels) and strains (right panels) in the rock matrix along the direction perpendicular to the fracture and for two different distances from the injection point ( $1 \mathrm{~m}$ and $10 \mathrm{~m}$ ). Bottom panels show the evolution of fluid temperature in the fracture (left) and fracture semi-aperture variation (right) along the fracture longitudinal direction. Colors correspond to different times, which are normalized with respect to the characteristic time $t_{c}=\theta^{2} D \tau^{2} / \pi / b^{2}$, where $\tau$ is the advective time to reach $x=1 \mathrm{~m}$. Results are normalized with respect to the injected temperature $T_{0}$. Flow velocity is assumed as constant, disregarding the effect of the aperture variation. Further details of the simulated example are given in the text.

\section{Comparison with classical solution for multiple station- ary and independent trajectories}

If thermal deformations are neglected, the fracture aperture and the velocity fields are constant in time. This implies that the trajectories are stationary for the duration of the heat injection. Under this circumstance it seems natural to extend the solution of Section 3.1 to the case of multiple trajectories, which is easily carried out by evaluating eq. (13) on each trajectory for a given time. This approach has been proposed by several authors for the case of pulse or continuous injection of solute tracer (e.g., Yamashita and Kimura, 1990; Cvetkovic et al., 1999; Tsang and Tsang, 2001; Liu et al., 2007) and recently applied to the case of heat injection (Gisladottir et al., 2016).

The adoption of this methodology implicitly entails neglecting the mutual effects between neighboring trajectories for all the duration of the injection. Thus, each trajectory is independent from the others and the fluid-rock heat exchange on one trajectory depends exclusively on the temperature evolution in time on the same trajectory. This is equivalent to consider that the heat exchanged by the generic particle $p$ at the time interval $i$ is

$$
J_{p}^{i}(\mathbf{x})=\sqrt{\frac{\kappa C_{m}}{\pi}}\left[\frac{T_{p}^{i}(\mathbf{x})}{\sqrt{\Delta t_{0}}}-\sum_{j=1}^{i-1} T_{p}^{j}(\mathbf{x}) f\left(t_{0}^{i}, t_{0}^{j}\right)\right] .
$$

Neglecting the effects of the lateral diffusion between neighboring trajectories is reasonable in the case of a pulse injection, because the time is too short for the heat to diffuse in the rock over the distance between the trajectories. The question is whether this assumption is valid for the case of a long continuous injection, which we analyze and discuss in this section.

To illustrate the effects of the assumption of independent trajectories when several particles crosses close locations, we perform the same analysis of Section 5.4, in which we have considered that the trajectories and the velocity field are stationary. We compare the results obtained with our approach with the ones obtained from the approach of Section 3.1 extended to multiple flow paths. The difference between the two methods is analyzed using the results evaluated with the contribution of all particles taken individually as the reference, in order to avoid 

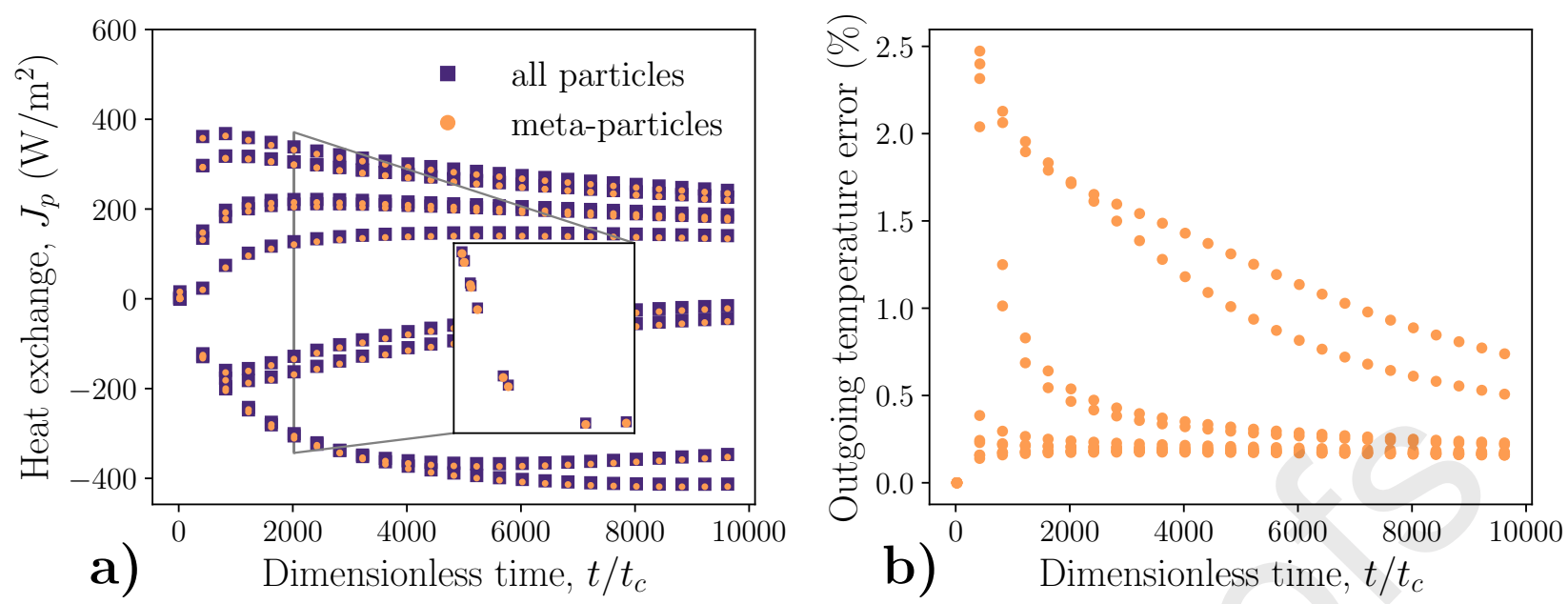

Outgoing temperature error $(\%)$
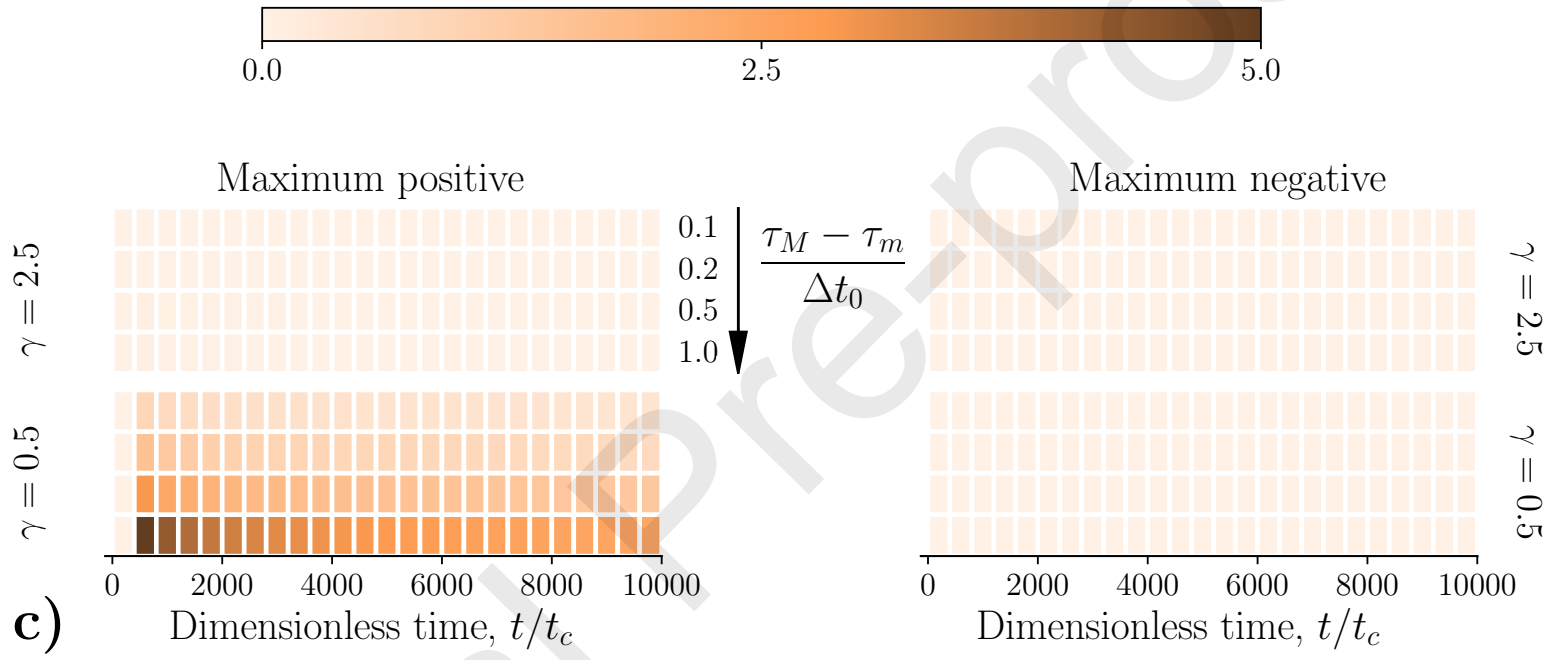

Figure 8: a) Diffusive fluid/rock heat flux, $J_{p}$, undergone by each particle at different times, evaluated considering the contribution of all particles taken individually (eq. (29), blue squares), and the contribution of all particles considering their average values of passage times and temperatures through a meta-particle concept (eq. (33), orange dots). Each marker corresponds to one particle. The same set of 10 particles arrive at the control location at each $t_{0}$ with stationary passage times $\tau$ following a power law pdf distribution and temperatures proportional to erfc $(\tau / \sqrt{t})$. b) Relative error in the estimation of the particle temperature after having exchanged $J_{p}$ with the rock for a $\Delta \tau=1 \mathrm{~s}$ when the meta-particle concept is used. c) Maximum values (averaged over 100 realizations) of positive and negative relative errors in the outgoing temperature when the meta-particle concept is used. Columns represent different observation times, rows represent different scenarios of values for the exponent of the passage time power law pdf distribution, $\gamma$, and for the time interval $\Delta t_{0}$. The results shown in the top panels correspond to one of the realizations of the case with $\gamma=0.5$ and $\Delta t_{0}=\tau_{M}-\tau_{m}$, represented in the last row of the colorplots. Time is normalized with respect to the characteristic time for the unitary $\Delta \tau$. For all plots, the writing frequency has been adjusted in order to show results at the same time for all cases. More details in Section 5.4 .

interference with the error associated with the meta-particle assumption.

Figure 9a shows that the assumption of independent flow paths leads to overestimate or underestimate $J_{p}$ (observe the green markers), because it is assumed to be exclusively a function of the temperature evolution on the particle trajectory. Therefore, $J_{p}$ is underestimated for the hottest particles and overestimated for the coldest ones. Observe that, when trajectories are not independent (blue markers), $J_{p}$ is negative for the slowest (and coldest) particles, because the rock matrix has increased its temperature so the diffusive heat flux is reverted (the cold particle can receive the heat released by the hot ones). This effect is not captured under the assumption of independent flow paths.
As a consequence of the large differences in $J_{p}$, the estimation of the exit temperature under the assumption of independent flow paths incurs substantial differences, which however decrease in time (Fig. 9b). The error is positive for most particles, but the negative error is larger that the positive, reflecting that the temperature is significantly underestimated for a few particles. This is a consequence of the assumed distribution of arrival time and temperature, with more particles with hot temperature than with low temperature. The heating of the rock matrix due to the hot particle contributions is not captured in the case of independent trajectories. As expected, the negative error is larger for the late arriving particles which are the coldest ones. Since the particle entrance temperature is proportional to the $\operatorname{erfc}(1 / \sqrt{t})$, the error decreases with time, as all the particles 


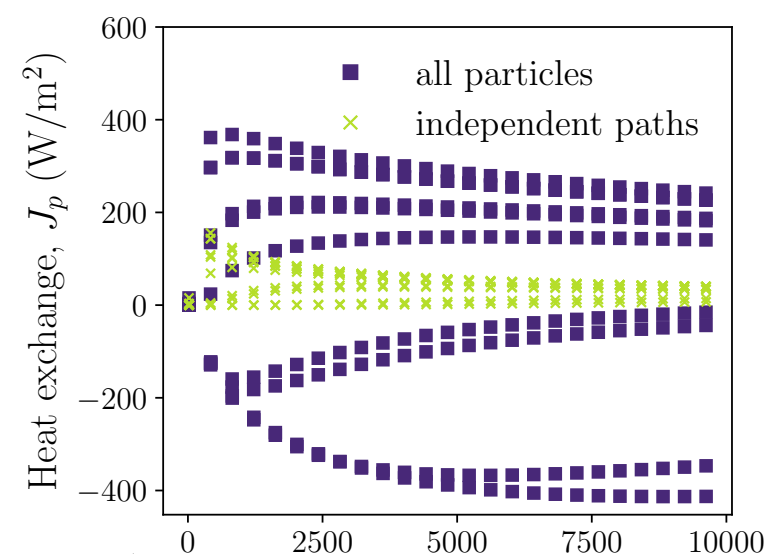

a) Dimensionless time, $t / t_{c}$

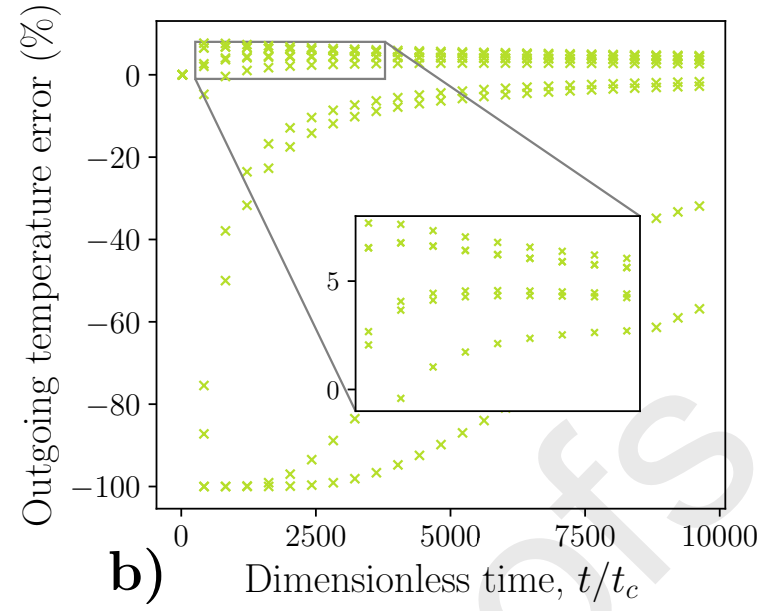

b) Dimensionless time, $t / t_{c}$

Outgoing temperature error $(\%)$

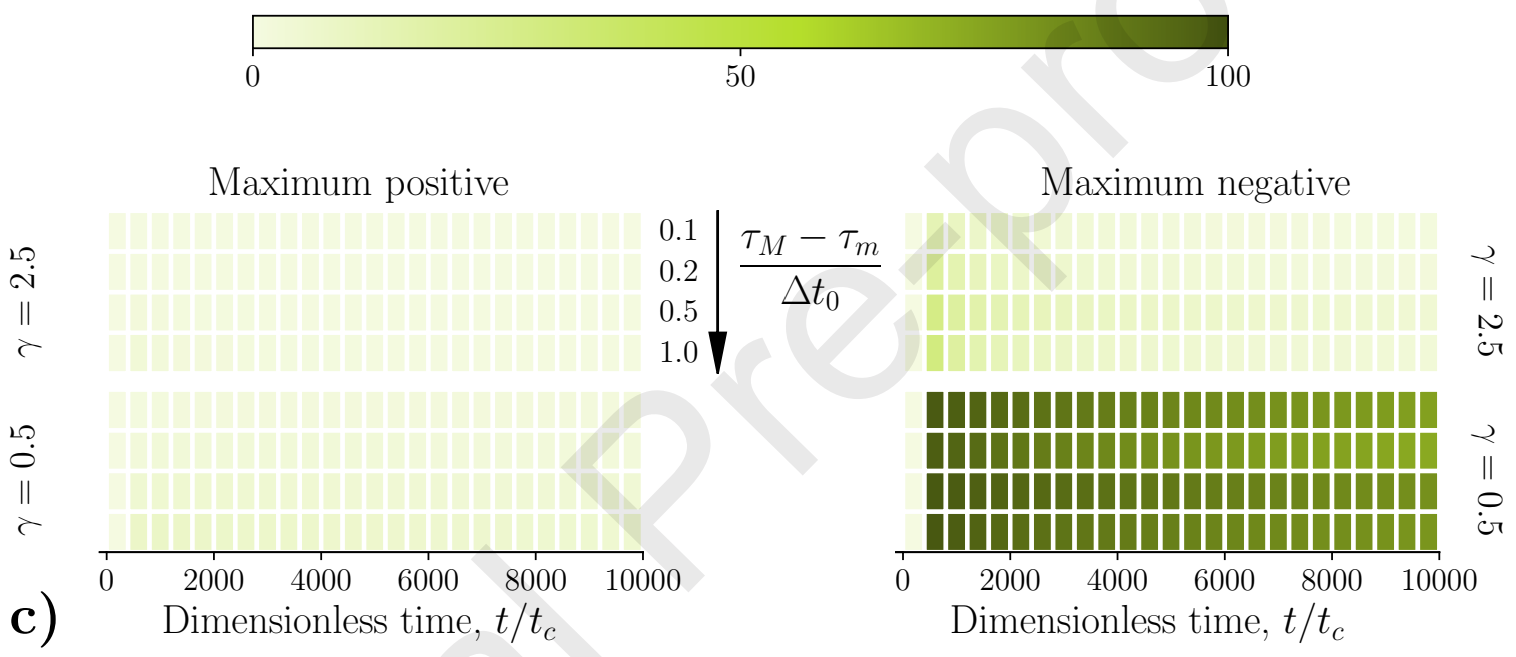

Figure 9: a) Diffusive fluid/rock heat flux, $J_{p}$, undergone by each particle at different times, evaluated considering the contribution of all particles taken individually (eqs. (28) and (29), blue squares), and the contribution of the single particle under the assumption of independent flow paths (eq. (38), green crosses). Each marker corresponds to one particle. The same set of 10 particles arrive at the control location at each $t_{0}$ with stationary passage times $\tau$ following a power law pdf distribution and temperatures proportional to $\operatorname{erfc}(\tau / \sqrt{t})$. b) Relative error in the estimation of the particle temperature after having exchanged $J_{p}$ with the rock for a $\Delta \tau=1 \mathrm{~s}$ when the assumption of independent flow paths is considered. c) Maximum values (averaged over 100 realizations) of positive and negative relative errors in the outgoing temperature when the assumption of independent flow paths is considered. Columns represent different observation times, rows represent different scenarios of values for the exponent of the passage time power law pdf distribution, $\gamma$, and for the time interval $\Delta t_{0}$. The results shown in the top panels correspond to one of the realizations of the case with $\gamma=0.5$ and $\Delta t_{0}=\tau_{M}-\tau_{m}$, represented in the last row of the colorplots. Time is normalized with respect to the characteristic time for the unitary $\Delta \tau$. For all plots, the writing frequency has been adjusted in order to show results at the same time for all cases. More details in Section 6.

tend to similar temperatures.

Results shown in Fig. 9a and b refer to the case with exponent of the passage times pdf distribution $\gamma$ equal to 0.5 , which corresponds to a case with high probability of having particles with large arrival time and low temperature. When many of such extremely slow particles exist, the classical method fails in estimating their temperatures and large relative errors are observed, with negative values up to $100 \%$ and positive values below $8 \%$. The behavior is however highly sensitive to the probability distribution exponent (Fig. 9c). In fact, the existence of a large number of particles with very different arrival times and temperatures constitutes a situation far from the independent trajectories assumption, while if many particles exhibit similar temperature and arrival time, the system approaches the behavior of a single trajectory. For this reason, the negative relative error is smaller than $30 \%$ when $\gamma=2.5$, indicating the two methods give similar results. Notice also that the difference between the two methods is almost insensitive to the time interval value. A slight increase of the error is observed for decreasing time interval, because for short time interval the particle temperatures are more often adjusted when based on the previous particle transition, while the time interval does not impact the results in the case of independent trajectories. As also commented in Section 5.4, the error decreases with time, coinciding with a reduction of the variability of particle arrival temperature. 
Despite the large difference observed for a few particle temperatures, the average temperature estimated over the ensemble of particles gives similar values with the two methods, because most particles have similar arrival times. This means that if the average temperature results are observed at a control point coinciding with the observation location here, we observe similar temperature whether we adopt the classical method or the new one. However, results evaluated at a downstream control section with one method or the other may differ if particles diverge as they bring a different temperature. It is clear that under certain conditions, the mutual effect between converging flow paths may affect the behavior and it should therefore be considered.

The analysis performed here and in Section 5.4 is intended to represent some of the most critical cases. In fact, trajectories with such heterogeneous characteristics converging into the same location reflects an extreme case that is hardly encountered in fracture networks, where adjacent trajectories typically exhibit similar arrival times. We further analyze and discuss the difference between the two methods by observing the result on a DFN in the next Section.

\section{Heat transport in a DFN}

To analyze the effects of the thermo-mechanical rock deformation on the heat transport in a fracture network, we compare the results from simulations with temperature-dependent fracture aperture and with constant fracture aperture. We also show the results evaluated with the classical approach of independent stationary trajectories (recall Section 6).

We consider a simple DFN composed by 10 fractures with equal fracture semi-aperture $b=10^{-4} \mathrm{~m}$, corresponding to a transmissivity approximately equal to $7 \times 10^{-6} \mathrm{~m}^{2} / \mathrm{s}$. The domain has a cubic volume of $100 \mathrm{~m}$ side. A temperature colder than the resident fluid by $50^{\circ} \mathrm{C}$ is injected from one section of the domain, on which we consider two scenarios of boundary conditions - one with constant inflow, the other with constant pressure - while constant pressure equal to zero is imposed at the opposite section. The constant inflow is set as equal to $3 \times 10^{-3} \mathrm{~m}^{3} / \mathrm{s}$, while the constant pressure is $5 \mathrm{MPa}-$ the two scenarios generating similar velocity fields. To perform the simulation, 500 particles are introduced in the system from the inlet at each time step. The resulting pdf distribution of particle travel times from inlet to outlet follows a power law with exponent slightly larger than 2 , and the maximum value is $2 \times 10^{4}$ s (Fig. 10, left panel). The characteristic time for the single jumps spans over 10 orders of magnitude ranging from $10^{-1} \mathrm{~s}$ to $10^{9} \mathrm{~s}$, with an average value of $3 \times 10^{6} \mathrm{~s}$ and a pdf distribution as shown in Fig. 10, right panel. According with these values, the time interval $\Delta t_{0}$ is set as equal to $1 \times 10^{6} \mathrm{~s}$, approximately 11 days. Different values of particle number and time interval have been also considered to ensure that results are independent on these factors (see Supplementary Information).

For illustrative purposes, we first consider injecting only four particles at each time step for the scenario with fixed inflow boundary condition at the inlet. Figure 11 shows the velocity field and trajectories of the four particles at the initial time and at time equal to $2 \times 10^{7} \mathrm{~s}$, for the case of temperature-dependent fracture aperture. As the velocity field evolve in time, particle trajectories migrate towards faster flow paths. These correspond to zones where the fracture aperture, and transmissivity, has increased as a consequence of the rock cooling and contraction (observe panel c). It is worth noting that, given the fixed inflow BC, flow velocity decreases everywhere in the network, but it is larger where the fracture aperture increases, which is different from what observed for the one-dimensional domain in Section 5.2. This different behavior is related to the increased complexity of the flow field.

The example above illustrates that the injection of a cold fluid, and the consequent rock contraction and fracture aperture increase, may cause the progressive concentration of the heat transport along preferential fast flow paths. To analyze the consequence of this behavior on the heat transport, we analyze the results of the simulation with the full set of 500 particles.

Figure 12 shows the temporal evolution of the temperature at the outlet, evaluated as the average temperature over the cross section. The comparison of the results for the two cases of constant and variable fracture aperture shows that heat transport is significantly accelerated when fracture aperture is temperaturedependent. This behavior is observed for both scenarios of boundary conditions, but the temperature profile is sharper when constant pressure is imposed at the inlet. This is not only a consequence of the non-locality of the velocity field under this boundary condition, as observed in Section 5.2, but it also depends on the particle injection mode. Since particles at the inlet are distributed proportionally to the flow velocity, at each time step more particles are injected in places with increased flow velocity. This mechanisms means that velocity will significantly increase on initially high-velocity paths, enlarging the difference between slow and fast paths.

When the imposed boundary condition is of constant inflow, particles are uniformly distributed over the inlet section. However, as observed in Fig. 11, particles tend to migrate towards faster regions, which promotes the advective transport resulting in a faster temperature response than in the case with constant aperture, where the trajectories are stationary. This migration explains the difference from what was observed for the one-dimensional domain, where the fracture with temperaturedependent aperture exhibits the same behavior than the fracture with constant aperture (compare Fig. 12 with Fig. 5).

In general, the dramatic acceleration of the advective transport is the primary reason for the faster heat transfer. There are however additional mechanisms involved. The passage through fractures with larger aperture implies a smaller diffusive temperature variation, because the fluid volume in contact with the rock surface is larger, or equivalently, the surface of diffusive exchange per fluid volume is smaller (recall eq. (3)). Moreover, the heat moves through rocks already affected by significant temperature variations, which reduces the temperature gradient at the fracture/rock interface, and therefore the heat exchange flux.

In the specific example here, all trajectories converge to the same fracture at the outlet (Fig. 11). It is therefore interesting to compare the results of the case with constant fracture 

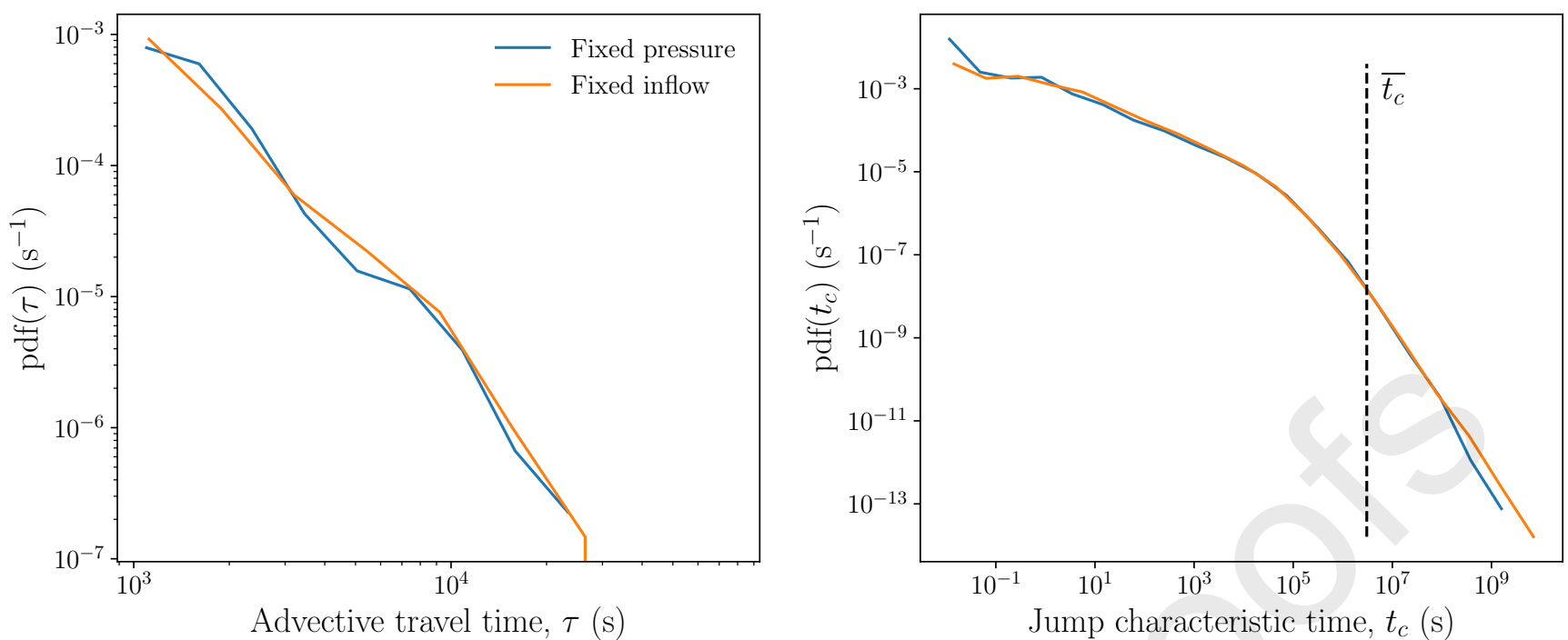

Figure 10: Probability density function (pdf) distributions of the time for the particles to travel from the inlet to the outlet (left) and of the characteristic time for the single jumps (right) for the two scenarios considered in the example DFN, i.e., with fixed pressure (blue) and fixed inflow (orange) at the inlet. The black dashed line indicates the average characteristic time. Values refer to the initial conditions of velocity and fracture aperture.
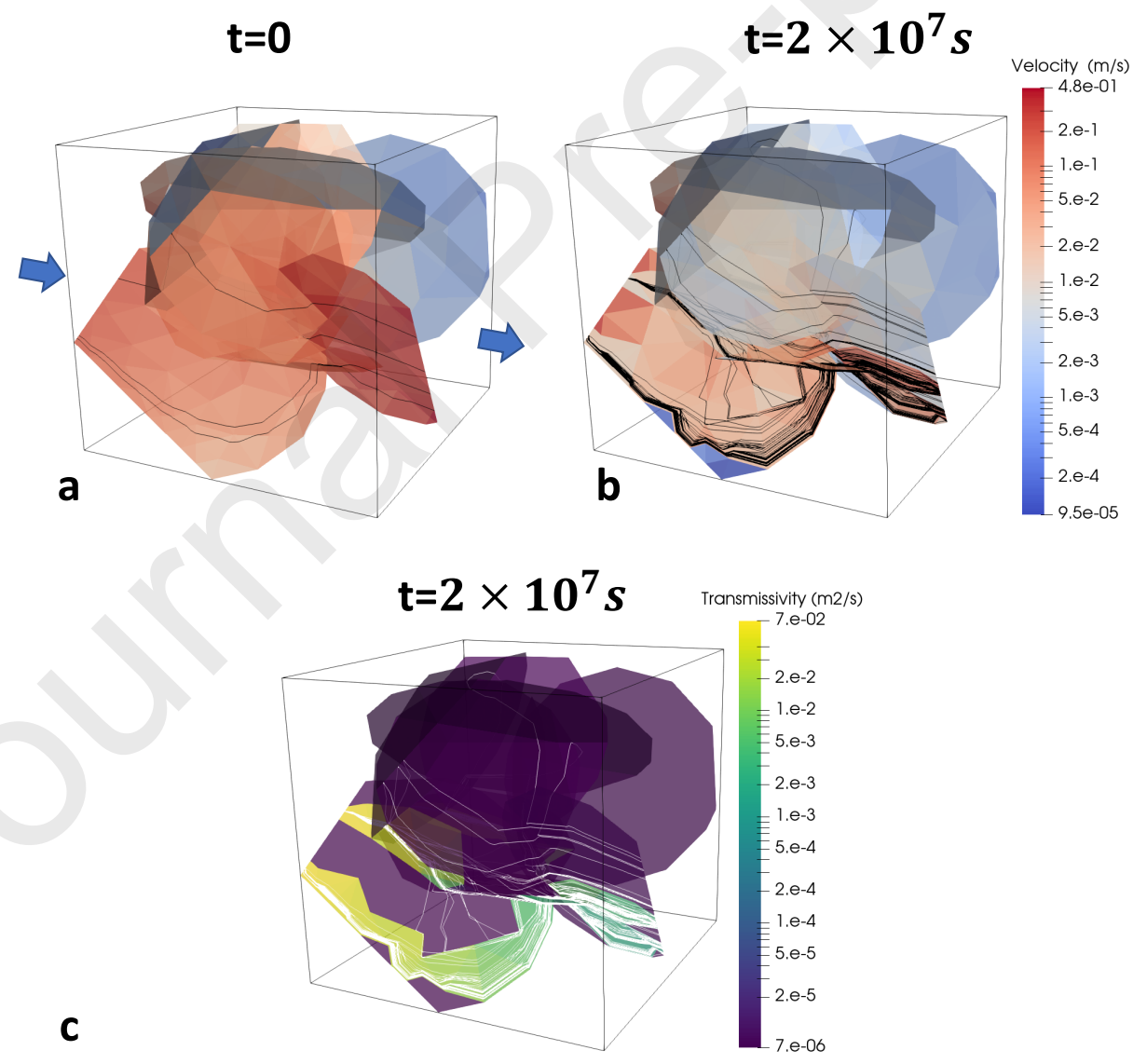

Figure 11: Velocity field at the initial time (a), velocity (b) and transmissivity (c) fields at time equal to $2 \times 10^{7} \mathrm{~s}$ in the example DFN with temperature-dependent fracture aperture under the continuous injection of 4 particles in the system. Black and white lines represent the trajectories of the particles injected until the represented time, where the color is solely chosen for visualization purposes. The arrows in (a) indicate the fluid inlet and outlet sections. 


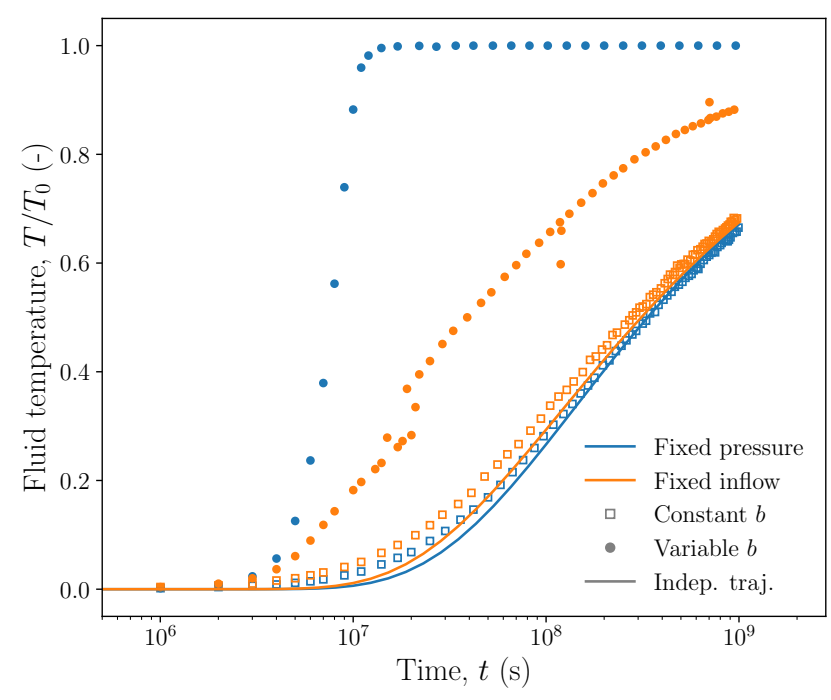

Figure 12: Temporal evolution of the average temperature at the outlet section of the example DFN for the two scenarios of constant inflow (orange) and constant pressure (blue) boundary conditions, and under the two cases of constant (empty square markers) and temperature-dependent (full dot markers) fracture aperture. Results evaluated with the assumption of independent trajectories are represented by the solid lines. Temperature is normalized with respect to the one imposed at the boundary. Plot-frequency is adjusted to improve visualization.

aperture against the results estimated by means of the classical method of independent trajectories. Figure 12 shows that the two methods give similar values of average temperature at the outlet. However, the individual particle temperature evolution (Fig. 13) shows that the assumption of independent trajectories implies a small overestimation and a large underestimation of the particle temperature, which is similar to what observed in Section 6. This occurs for a small number of particles, and therefore the temperature estimated as the average over all the particle temperatures is similar. Nevertheless, the error may propagate if particles diverge down-stream, which will be the focus of future investigations.

\section{Discussion}

The formulation presented above is ideally conceived to modeling heat transport with thermo-mechanical effects due to continuous non-isothermal injection into a complex fracture system, such as in the case of geothermal technologies.

Eulerian numerical models, such as finite element or finite difference methods, typically suffer from numerical instability in solving transport problems, especially when the advective front is sharp. Lagrangian methods overcome this problem, but in general they do not allow taking into account variability in time of the parameters. The hybrid approach proposed in this paper provides a stable algorithm for the transport advectivediffusive problem while keeping the variable-in-time nature of the parameters. The adoption of a grid-defined memory diffusive heat exchange allows us to deal with trajectories that change in time because of the thermal deformation of the host

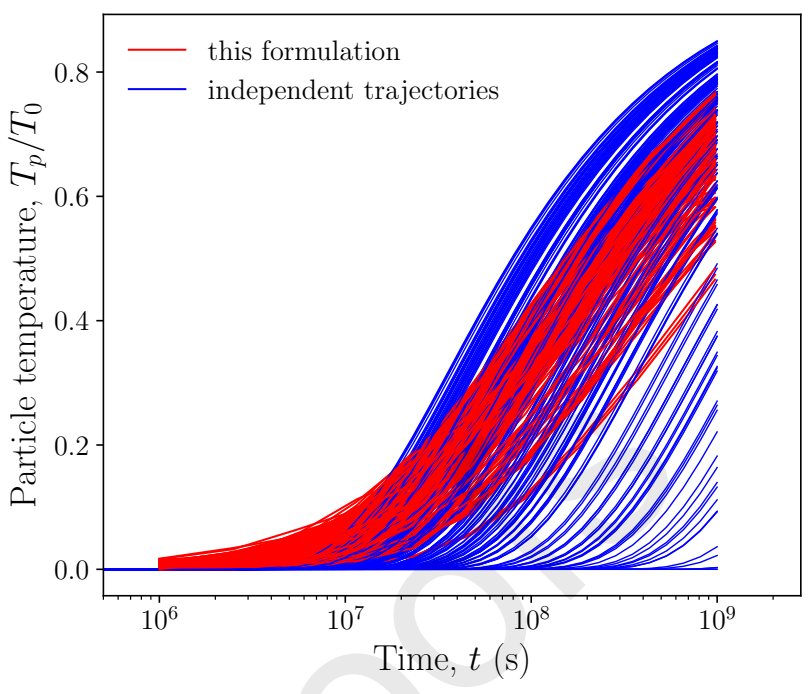

Figure 13: Temporal evolution of the particle temperature in the example DFN, evaluated by means of the formulation presented here under the condition of constant fracture aperture (red lines) and by means of the classical approach with independent trajectories (blue lines). For aesthetic reasons, we only show the evolution for 200 of the 500 injected particles, but the behavior of the full set of particles is not dissimilar. Temperature is normalized with respect to the one imposed at the boundary.

rock. The analytic solution of both the diffusive and the mechanical part of the problem sensibly reduces the computational cost. These remarkable advantages imply the cost of sacrificing some of the complexities of the problem.

Injection of cold fluids in fractured media causes an increase in pore pressure and a decrease in temperature, both processes leading to an increase in the fracture aperture (Koh et al., 2011; Ghassemi and Tao, 2016). The two processes act on very different time scales, with heat transfer occurring at very large time-scale compared to the pressure diffusion (De Simone et al., 2017). Building on this concept, we disregard the HM fracture aperture variation, and we concentrate on the solely TM elastic deformations. This conceptually corresponds to analyzing the long-term effects, after the early-time pressure-driven deformations have occurred. At the same time, we disregard some complexity intrinsic to the coupled THM process, like the transient undrained increase in pore pressure consequent to the thermal deformation of low transmissivity saturated rocks. Since we assume impermeable rock mass, fluid pressure leak-off and the associated hydro-mechanical rock deformation are also disregarded. These effects may be significant in certain situations, whose modeling requires the use of fully coupled THM simulations (Rutqvist et al., 2002). Variation of fluid density and viscosity due to temperature variations has not been considered, but it can be easily incorporated as a feature of the velocity field update.

We also make the assumption that the matrix is infinite in the direction perpendicular to the fracture walls. This is of course an over-simplification, whose effect is twofold. On the one hand, diffusion through the matrix may lead to temperature variations in fractures not directly involved by advective 
processes, or to faster temperature variations in fractures where the advective transport is slow. Thus, neglecting the finite size of the rock may lead to overestimations of the transfer times (Roubinet et al., 2010). On the other hand, the infinite matrix assumption does not consider that the deformation of the rock matrix may affect the aperture of other fractures. Both effects take place for time larger than the diffusive time over the distance between two fractures $d, t_{D}=d^{2} / D$, which is approximately 11 days if $d=1 \mathrm{~m}$, and 3 years if $d=10 \mathrm{~m}$. This time constitutes a threshold for the validity of our formulation and relaxing this limitation will be object of future work.

With respect to the heat transport within the fractures, we assume the parallel plate model with transverse diffusion into the matrix. However, field experiments have shown that rough fractures often exhibit flow concentration into channels, which have diffusive interfaces more similar to tubes than to planes (Klepikova et al., 2016; de La Bernardie et al., 2018). This leads to a faster heat transfer and a different scaling in time with respect to the parallel plate model. The incorporation of these features is beyond the scope of this paper, but it will be the object of future developments.

Although no explicit term relative to lateral diffusion is included in our formulation (Section 2.2), its effects are somehow modeled by means of the memory diffusive heat exchange, whose values are updated at each time step as the result of a temperature homogenization over each mesh element (recall Section 4). This operation may appear mesh size-dependent. However, a necessary condition to get representative results in any particle-tracking simulation is considering a large number of particles. When a sufficient large number of particles is introduced in the system, neighboring mesh elements undergo similar temperature variations, thus the mesh size does not have great impacts on the resulting temperature field. For the same reason, we do not explicitly introduce any lateral diffusion between mesh elements.

The operation of rock temperature homogenization over the mesh grid marks the difference with respect to the classical method, where trajectories are assumed as independent and the fracture-rock heat exchange is independently evaluated on each trajectory. The assumption of independent trajectories may be limiting in cases where different trajectories cross neighboring locations at different times, because previous variations of the rock temperature due to the contribution of other particles are disregarded. This may lead to an overestimation or underestimation of $J$, which is significant if the particles converge to the same mesh element with very different travel times, a condition that is scarcely encountered in most DFN (Section 6).

Similar to any other numerical method based on time increments, the accuracy and efficiency of the here proposed method is affected by the adopted time interval. As discussed in Section 4 , the adoption of a time interval larger than the advective time for traveling from the inlet to the outlet is convenient to prevent the simultaneous presence of multiple particle sets in the system, and the associated memory demand. Time intervals larger than the advective travel time variance are convenient to prevent large errors associated with the meta-particle assumption (Section 5.4). Finally, a large time interval helps in speed- ing up the simulation time. On the other hand, we have shown that time intervals much larger than the problem characteristic time may give less accurate results (see Section 5.1). It is clear that an evaluation of the optimal time interval is required previous to performing the simulation, in order to find a value large enough to ensure efficiency while preserving accuracy. Defining a time interval that meet the above discussed requirements is, however, not difficult provided the range of values that define the problem. For the sake of simplicity, consider the time for a particle to travel a distance $L$ as equal to the number of jumps over the mesh elements, $m$, multiplied by the average time for the jump, $\overline{\Delta \tau}$. We seek for a $\Delta t_{0}$ larger than the total travel time but smaller than the characteristic time for the jump, i.e., $\Delta t_{0}>m \overline{\Delta \tau}$ and $\Delta t_{0}<D \overline{\Delta \tau}^{2} / \pi / b^{2}$, where $D$ is approximately $10^{-6} \mathrm{~m}^{2} / \mathrm{s}$ for rocks and $b$ typically ranges between $10^{-6}$ and $10^{-2} \mathrm{~m}^{2}$. For example, if the average value of $b$ is $10^{-4} \mathrm{~m}^{2}$, the average velocity is $10^{-3} \mathrm{~m} / \mathrm{s}, L=100$ and the average mesh size equal to $1 \mathrm{~m}$, then $m=100, \overline{\Delta \tau}=10^{3} \mathrm{~s}$ and the optimum $\Delta t_{0}$ is between $10^{5} \mathrm{~s}$ and $10^{7} \mathrm{~s}$. Nevertheless, the variability of the characteristic time for the single jump may be large (Fig. 10), as it is defined by the spatial variability of fracture aperture, flow velocity and jump length. The latter is related with the mesh size, which can be highly variable in networks with many intersections and large variability of the fracture size. The jump length is very small when the trajectory crosses small mesh elements or mesh element corners, which requires reducing the time interval. The consequent increase of computational time may be significant, especially in the case of complex networks with large number of fractures and particles. Improving this aspect is crucial to reduce the computational time, and it will be the topic of future work.

\section{Conclusions}

We have proposed and validated a new formulation to simulate advective-diffusive heat transport in heterogeneous threedimensional fracture networks with thermo-mechanical fracture aperture and flow velocity variation.

The method is conceived to be implemented into a particletracking approach where each particle holds a temperature that changes because of the diffusive exchange with the rock matrix. The diffusive flux and the associate thermo-mechanical rock deformation are estimated adopting a semi-analytic scheme with time discretization. This allows to consider trajectory variations due to thermo-mechanical deformations. To our best knowledge, the current study represents the first attempt to incorporate the thermo-mechanical behavior of the rock matrix into a particle-tracking method. Further work will entail overcoming the limiting assumption of infinite rock matrix and perfectly planar fractures, as well as improving the time interval control to ensure accuracy.

Although subject to several simplifications, the methodology enables investigating the reservoir long-term TM behavior while keeping the computational effort within reasonable values. The computation time is in fact of the order of minuteshours compared with the hours-days required by fully coupled 
methods. Our formulation is therefore ideal for stochastic modeling, which allows exploring the uncertainty in cases when the site characterization is poor. For this reason, this method represents a useful tool for the identification of potential bottlenecks, the design of optimal strategies for heat recovery and the management of geothermal system exploitation.

\section{Acknowledgments}

The authors acknowledge financial support from the SADGEOTHERM project (SAD18007) funded by the Brittany Region under the SAD2018 framework (France) and from the ANR-17-LCV2-0012 eLabo project funded by the Agence Nationale de la Recherche ANR (France). All materials, including scripts, input and output files, are available at the repository http://doi.org/10.5281/zenodo.5139985. The DFN.lab web page is https://fractorylab.org/. Details on the use and availability of the software are available at https://gitlab.com/fractorylab/dfnlab.

\section{Appendix A. Solution to constant temperature boundary condition in single flow path with constant fracture aperture and flow velocity}

Laplace inversion of eq. (5), with zero temperature initial conditions, gives

$$
s \hat{T_{m}}=D \frac{\mathrm{d}^{2} \hat{T_{m}}}{\mathrm{~d} z^{2}},
$$

where the hat denotes the functions in Laplace space, $s$ is the Laplace variable and $D=\kappa / C_{m}$ is the matrix thermal diffusion. Solution of this equation in the Laplace domain is

$$
\hat{T_{m}}(\ell, z, s)=A_{1} \exp [-\sqrt{(s / D)}(z-b)]+A_{2} \exp [\sqrt{(s / D)}(z-b)] .
$$

Constant $A_{2}$ must be 0 to ensure the $\mathrm{BC}$ at $z=\infty$, whereas $A_{1}$ is equal to $\hat{T}(\ell, s)$ to ensure the $\mathrm{BC}$ at the matrix/fracture interface (recall eqs. (7)). By substituting eq. (A.2) into eq. (4) , we obtain an expression for the diffusive flux in the Laplace domain

$$
\hat{J}(\ell, t)=\sqrt{\kappa C_{m} s} \hat{T} .
$$

This latter can be substituted into the Laplace transform of eq. (12), with zero temperature initial conditions, which gives

$$
b s \hat{T}+\frac{\mathrm{d} b v \hat{T}_{f}}{\mathrm{~d} \ell}=-\theta \sqrt{D s} \hat{T}
$$

where $\theta$ is the ratio of matrix to fracture heat capacity, i.e., $\theta=$ $C_{m} / C$. By assuming

$$
\mathrm{d} B=\frac{\mathrm{d} \ell}{b(\ell) v(\ell)}
$$

we can rearrange eq. (A.4) into

$$
\frac{\mathrm{d} \hat{T}}{\hat{T}}=-(b s+\theta \sqrt{D s}) \mathrm{d} B
$$

which solution is

$$
\hat{T}(\ell, s)=A_{3} \exp \left[-\int_{0}^{B}(b s+\theta \sqrt{D s}) \mathrm{d} B^{\prime}\right] .
$$

The constant $A_{3}$ is found by imposing the boundary condition for $T$ at $\ell=0$, which for continuous injection and in the Laplace domain is $\hat{T}(0, s)=T_{0} / s$.

The solution in the Laplace domain is therefore

$$
\hat{T}(\ell, s)=\frac{T_{0}}{s} \exp \left[-s \int_{0}^{\ell} \frac{\mathrm{d} \ell^{\prime}}{v}-\theta \sqrt{D s} \int_{0}^{\ell} \frac{\mathrm{d} \ell^{\prime}}{b v}\right]
$$

where we have made use of eq. (A.5) and we have assumed uniform $D$ for simplicity.

Inversion of this equation gives the solution for the temperature in the fracture in the time domain (eq. (13)). Substitution of eq. (A.8) into eq. (A.2) yields

$$
\hat{T_{m}}(\ell, z, s)=\frac{T_{0}}{s} \exp \left[-s \int_{0}^{\ell} \frac{\mathrm{d} \ell^{\prime}}{v}-\theta \sqrt{D s}\left(\int_{0}^{\ell} \frac{\mathrm{d} \ell^{\prime}}{b v}+\frac{z-b}{\theta D}\right)\right]
$$

whose expression in the time domain is given in eq. (14).

\section{Appendix B. General expression for rock matrix tempera- ture $T_{m}$ and diffusive heat flux $J$}

Diffusion in the matrix is solved in the Laplace space as in Appendix Appendix A. Inversion of the Laplace solution (eq. (A.2)) returns that the temperature in the matrix is the convolution integral of the temperature at the fracture/matrix interface and the diffusive one-dimensional response to the pulse injection, $\psi(z, t)$

$$
\begin{aligned}
T_{m}(\ell, z, t) & =\left[T_{m}(\ell, b, t) * \psi(z, t)\right](t) \\
& =\int_{0}^{t} T_{m}\left(\ell, b, t^{\prime}\right) \psi\left(z, t-t^{\prime}\right) \mathrm{d} t^{\prime}
\end{aligned}
$$

where

$$
\psi(z, t)=\frac{z-b}{2 \sqrt{\pi D} t^{3 / 2}} \exp \left(-\frac{(z-b)^{2}}{4 D t}\right) .
$$

Substitution of eq. (B.1) into eq. (4) and differentiation under the integral sign returns

$$
J(\ell, t)=-\left.\kappa \int_{0}^{t} T_{m}\left(\ell, b, t^{\prime}\right) \frac{\partial \psi\left(z, t-t^{\prime}\right)}{\partial z} \mathrm{~d} t^{\prime}\right|_{z=b}
$$

which after differentiation reads

$$
\begin{aligned}
J(\ell, t) & =-\kappa \int_{0}^{t} \frac{T_{m}\left(\ell, b, t^{\prime}\right)}{2 \sqrt{\pi D}\left(t-t^{\prime}\right)^{3 / 2}} \times \\
& \left.\exp \left(-\frac{(z-b)^{2}}{4 D\left(t-t^{\prime}\right)}\right)\left(1-\frac{(z-b)^{2}}{2 D\left(t-t^{\prime}\right)}\right) \mathrm{d} t^{\prime}\right|_{z=b} .
\end{aligned}
$$

Note that if $T_{m}\left(\ell, b, t^{\prime}\right)$ is constant for an interval of time between $t_{1}$ and $t_{2}$, then the heat flux corresponding to the interval is

$$
J(\ell, t)_{\left[t_{1}, t_{2}\right]}=-\sqrt{\frac{\kappa C_{m}}{\pi}} T_{m}(\ell, b)_{\left[t_{1}, t_{2}\right]}\left(\frac{1}{\sqrt{t-t_{2}}}-\frac{1}{\sqrt{t-t_{1}}}\right),
$$


whereas if the interval goes from $t_{2}$ to $t$, it is

$$
J(\ell, t)_{\left[t_{2}, t\right]}=\sqrt{\frac{\kappa C_{m}}{\pi}} \frac{T_{m}(\ell, b)_{\left[t_{1}, t_{2}\right]}}{\sqrt{t-t_{2}}} .
$$

The same results can be obtained if the differentiation is not performed under the integral sign, as well as directly from the Laplace inversion of eq. (A.3), although the inversion of $\sqrt{s}$ requires invoking numerical inversion or the pseudo-transform concept (Abate and Valkó, 2004).

\section{References}

Abate, J., Valkó, P.P., 2004. Multi-precision laplace transform inversion. International Journal for Numerical Methods in Engineering 60, 979-993. doi:10.1002/nme.995.

Baria, R., Baumgärtner, J., Rummel, F., Pine, R.J., Sato, Y., 1999. HDR/HWR reservoirs: concepts, understanding and creation. Geothermics 28, 533-552. doi:10.1016/s0375-6505(99)00045-0.

Becker, M.W., Shapiro, A.M., 2003. Interpreting tracer breakthrough tailing from different forced-gradient tracer experiment configurations in fractured bedrock. Water Resources Research 39. doi:10.1029/2001wr001190.

Bonnet, E., Bour, O., Odling, N.E., Davy, P., Main, I., Cowie, P., Berkowitz, B., 2001. Scaling of fracture systems in geological media. Reviews of Geophysics 39, 347-383. doi:10.1029/1999rg000074.

Bour, O., Davy, P., 1997. Connectivity of random fault networks following a power law fault length distribution. Water Resources Research 33, 15671583. doi:10.1029/96wr00433.

Bour, O., Davy, P., 1999. Clustering and size distributions of fault patterns: Theory and measurements. Geophysical Research Letters 26, 2001-2004. doi:10.1029/1999g1900419.

Bruel, D., 2002. Impact of induced thermal stresses during circulation tests in an engineered fractured geothermal reservoir: Example of the soultz-sousforêts european hot fractured rock geothermal project, rhine graben, france. Oil \& Gas Science and Technology 57, 459-470. doi:10.2516/ogst:2002030.

Carrera, J., Sánchez-Vila, X., Benet, I., Medina, A., Galarza, G., Guimerà, J., 1998. On matrix diffusion: formulations, solution methods and qualitative effects. Hydrogeology Journal 6, 178-190.

Cvetkovic, V., Selroos, J.O., Cheng, H., 1999. Transport of reactive tracers in rock fractures. Journal of Fluid Mechanics 378, 335-356. doi:10.1017/s0022112098003450.

Dagan, G., Bresler, E., 1979. Solute dispersion in unsaturated heterogeneous soil at field scale: I. theory. Soil Science Society of America Journal 43, 461-467. doi:10.2136/sssaj1979.03615995004300030008x

Dagan, G., Cvetkovic, V., Shapiro, A., 1992. A solute flux approach to transport in heterogeneous formations: 1. the general framework. Water Resources Research 28, 1369-1376. doi:10.1029/91wr03086.

Davy, P., Goc, R.L., Darcel, C., Bour, O., de Dreuzy, J.R., Munier, R., 2010. A likely universal model of fracture scaling and its consequence for crustal hydromechanics. Journal of Geophysical Research 115. doi:10.1029/2009jb007043.

De Simone, S., Carrera, J., Gómez-Castro, B.M., 2017. A practical solution to the mechanical perturbations induced by non-isothermal injection into a permeable medium. International Journal of Rock Mechanics and Mining Sciences 91, 7-17. doi:10.1016/j.ijrmms.2016.11.001.

de Dreuzy, J.R., Davy, P., Bour, O., 2001. Hydraulic properties of twodimensional random fracture networks following a power law length distribution: 2. permeability of networks based on lognormal distribution of apertures. Water Resources Research 37, 2079-2095. doi:10.1029/2001wr900010.

Flemisch, B., Berre, I., Boon, W., Fumagalli, A., Schwenck, N., Scotti, A., Stefansson, I., Tatomir, A., 2018. Benchmarks for single-phase flow in fractured porous media. Advances in Water Resources 111, 239-258. doi:10.1016/j.advwatres.2017.10.036.

Frampton, A., Cvetkovic, V., 2011. Numerical and analytical modeling of advective travel times in realistic three-dimensional fracture networks. Water Resources Research 47. doi:10.1029/2010wr009290.
Ghassemi, A., Tao, Q., 2016. Thermo-poroelastic effects on reservoir seismicity and permeability change. Geothermics 63, 210-224. doi:10.1016/j.geothermics.2016.02.006.

Giardini, D., 2009. Geothermal quake risks must be faced. Nature 462, 848849. doi: $10.1038 / 462848$ a.

Gisladottir, V.R., Roubinet, D., Tartakovsky, D.M., 2016. Particle methods for heat transfer in fractured media. Transport in Porous Media 115, 311-326. doi:10.1007/s11242-016-0755-2.

Hu, M., Rutqvist, J., Wang, Y., 2017. A numerical manifold method model for analyzing fully coupled hydro-mechanical processes in porous rock masses with discrete fractures. Advances in Water Resources 102, 111-126. doi:10.1016/j.advwatres.2017.02.007.

Hyman, J.D., Dentz, M., Hagberg, A., Kang, P.K., 2019. Linking structural and transport properties in three-dimensional fracture networks. Journal of Geophysical Research: Solid Earth 124, 1185-1204. doi: $10.1029 / 2018 \mathrm{jb} 016553$.

Jing, L., 2003. A review of techniques, advances and outstanding issues in numerical modelling for rock mechanics and rock engineering. International Journal of Rock Mechanics and Mining Sciences 40, 283-353. doi:10.1016/s1365-1609(03)00013-3.

Jung, R., 2013. EGS - goodbye or back to the future 95, in: Effective and Sustainable Hydraulic Fracturing. InTech. doi:10.5772/56458.

Klepikova, M.V., Borgne, T.L., Bour, O., Dentz, M., Hochreutener, R., Lavenant, N., 2016. Heat as a tracer for understanding transport processes in fractured media: Theory and field assessment from multiscale thermal push-pull tracer tests. Water Resources Research 52, 5442-5457. doi:10.1002/2016wr018789.

Koh, J., Roshan, H., Rahman, S.S., 2011. A numerical study on the long term thermo-poroelastic effects of cold water injection into naturally fractured geothermal reservoirs. Computers and Geotechnics 38, 669-682. doi:10.1016/j.compgeo.2011.03.007.

Konikow, L.F., Bredehoeft, J.D., 1978. Computer model of two-dimensional solute transport and dispersion in ground water. US Government Printing Office Washington, DC 7.

de La Bernardie, J., Bour, O., Borgne, T.L., Guihéneuf, N., Chatton, E., Labasque, T., Lay, H.L., Gerard, M.F., 2018. Thermal attenuation and lag time in fractured rock: Theory and field measurements from joint heat and solute tracer tests. Water Resources Research 54. doi:10.1029/2018wr023199.

Le Goc, R., Pinier, B., Darcel, C., Lavoine, E., Doolaeghe, D., Simone, S.D., de Dreuzy, J.R., Davy., P., 2019. Dfn.lab: Software platform for discrete fracture network models. AGU Fall Meeting. San Francisco, CA, USA, 2019 .

Lei, Q., Latham, J.P., Tsang, C.F., 2017. The use of discrete fracture networks for modelling coupled geomechanical and hydrological behaviour of fractured rocks. Computers and Geotechnics 85, 151-176. doi:10.1016/j.compgeo.2016.12.024.

Liu, H., Zhang, Y., Zhou, Q., Molz, F., 2007. An interpretation of potential scale dependence of the effective matrix diffusion coefficient. Journal of Contaminant Hydrology 90, 41-57. doi:10.1016/j.jconhyd.2006.09.006.

Matringe, S.F., Juanes, R., Tchelepi, H.A., 2006. Robust streamline tracing for the simulation of porous media flow on general triangular and quadrilateral grids. Journal of Computational Physics 219, 992-1012. doi:10.1016/j.jcp.2006.07.004.

McDermott, C.I., Randriamanjatosoa, A.R., Tenzer, H., Kolditz, O., 2006. Simulation of heat extraction from crystalline rocks: The influence of coupled processes on differential reservoir cooling. Geothermics 35, 321-344. doi:10.1016/j.geothermics.2006.05.002.

Moreno, L., Neretnieks, I., 1993. Flow and nuclide transport in fractured media: The importance of the flow-wetted surface for radionuclide migration. Journal of Contaminant Hydrology 13, 49-71. doi:10.1016/01697722(93)90050-3.

Odsæter, L.H., Kvamsdal, T., Larson, M.G., 2019. A simple embedded discrete fracture-matrix model for a coupled flow and transport problem in porous media. Computer Methods in Applied Mechanics and Engineering 343, 572-601. doi:10.1016/j.cma.2018.09.003.

Olivella, S., Gens, A., Carrera, J., Alonso, E., 1996. Numerical formulation for a simulator (CODE_BRIGHT) for the coupled analysis of saline media. Engineering Computations 13, 87-112. doi:10.1108/02644409610151575.

Painter, S., Cvetkovic, V., 2005. Upscaling discrete fracture network simulations: An alternative to continuum transport models. Water Resources 
Research 41. doi:10.1029/2004wr003682.

Pinier, B., Le Goc, R., Davy, P., 2021. Computation of flow and transport properties on large $3 \mathrm{~d}$ heterogeneous stochastic and semi-deterministic fractured media with the platform dfn.lab. in prep. .

Roubinet, D., Liu, H.H., de Dreuzy, J.R., 2010. A new particle-tracking approach to simulating transport in heterogeneous fractured porous media. Water Resources Research 46. doi:10.1029/2010wr009371.

Rutqvist, J., Wu, Y.S., Tsang, C.F., Bodvarsson, G., 2002. A modeling approach for analysis of coupled multiphase fluid flow, heat transfer, and deformation in fractured porous rock. International Journal of Rock Mechanics and Mining Sciences 39, 429-442. doi:10.1016/s1365-1609(02)00022-9.

Tang, D.H., Frind, E.O., Sudicky, E.A., 1981. Contaminant transport in fractured porous media: Analytical solution for a single fracture. Water Resources Research 17, 555-564. doi:10.1029/wr017i003p00555.

Tester, J.W., Anderson, B.J., Batchelor, A.S., Blackwell, D.D., DiPippo, R. Drake, E.M., Garnish, J., Livesay, B., Moore, M.C., Nichols, K., Petty, S., Toksoz, M.N., Veatch, R.W., Baria, R., Augustine, C., Murphy, E., Negraru, P., Richards, M., 2007. Impact of enhanced geothermal systems on US energy supply in the twenty-first century. Philosophical Transactions of the Royal Society A: Mathematical, Physical and Engineering Sciences 365, 1057-1094. doi:10.1098/rsta.2006.1964.

Timoshenko, S.P., Goodier, J., 1951. Theory of elasticity. McGraw-Hill. New York 56, 308-308. doi:10.1017/s036839310012471x.

Tsang, Y.W., Tsang, C.F., 2001. A particle-tracking method for advective transport in fractures with diffusion into finite matrix blocks. Water Resources Research 37, 831-835. doi:10.1029/2000wr900367.

Yamashita, R., Kimura, H., 1990. Particle-tracking technique for nuclide decay chain transport in fractured porous media. Journal of Nuclear Science and Technology 27, 1041-1049. doi:10.1080/18811248.1990.9731289. 